\title{
The RGB tip of galactic globular clusters and the revision of the axion-electron coupling bound
}

\author{
O. Straniero ${ }^{1,2}$, C. Pallanca ${ }^{3,4}$, E. Dalessandro 4 , I. Domínguez ${ }^{5}$, F. R. Ferraro ${ }^{3,4}$, M. Giannotti ${ }^{6}$, \\ A. Mirizzi ${ }^{7,8}$, and L. Piersanti ${ }^{1}$ \\ 1 INAF, Osservatorio d'Abruzzo,Via M. Maggini, 64100 Teramo, Italy \\ e-mail: oscar.straniero@inaf.it \\ 2 INFN, Laboratori Nazionali del Gran Sasso, Assergi, Italy \\ 3 Dipartimento di Fisica e Astronomia, Università di Bologna, Via Gobetti 93/2, 40129 Bologna, Italy \\ 4 INAF, Osservatorio di Astrofisica e Scienza dello Spazio di Bologna, Via Gobetti 93/3, 40129 Bologna, Italy \\ 5 Departamento de Fisíca Teoríca y del Cosmos, Universidad de Granada, 18071 Granada, Spain \\ 6 Physical Sciences, Barry University, 11300 NE 2nd Ave., Miami Shores 33161, USA \\ 7 Dipartemento Interateneo di Fisica "Michelangelo Merlin", Via Amendola 173, 70126 Bari, Italy \\ 8 INFN, Sezione di Bari, Via Orabona 4, 70126 Bari, Italy
}

Received 27 June 2020 / Accepted 6 October 2020

\begin{abstract}
Context. The production of neutrinos by plasma oscillations is the most important energy sink process operating in the degenerate core of low-mass red giant stars. This process counterbalances the release of energy induced by nuclear reactions and gravitational contraction, and determines the luminosity attained by a star at the moment of the He ignition. This occurrence coincides with the tip of the red giant branch (RGB), whose luminosity is extensively used as a calibrated standard candle in several cosmological studies. Aims. We aim to investigate the possible activation of additional energy sink mechanisms, as predicted by many extensions of the socalled Standard Model. In particular, our objective is to test the possible production of axions or axion-like particles, mainly through their coupling with electrons.

Methods. By combining Hubble Space Telescope and ground-based optical and near-infrared photometric samples, we derived the RGB tip absolute magnitude of 22 galactic globular clusters (GGCs). The effects of varying the distance and the metallicity scales were also investigated. Then we compared the observed tip luminosities with those predicted by state-of-the-art stellar models that include the energy loss due to the axion production in the degenerate core of red giant stars.

Results. We find that theoretical predictions including only the energy loss by plasma neutrinos are, in general, in good agreement with the observed tip bolometric magnitudes, even though the latter are $\sim 0.04$ mag brighter on average. This small shift may be the result of systematic errors affecting the evaluation of the RGB tip bolometric magnitudes, or, alternatively, it could be ascribed to an axion-electron coupling causing a non-negligible thermal production of axions. In order to estimate the strength of this possible axion sink, we performed a cumulative likelihood analysis using the RGB tips of the whole set of 22 GGCs. All the possible sources of uncertainties affecting both the measured bolometric magnitudes and the corresponding theoretical predictions were carefully considered. As a result, we find that the value of the axion-electron coupling parameter that maximizes the likelihood probability is $g_{a e} / 10^{-13} \sim 0.60_{-0.58}^{+0.32}$. This hint is valid, however, if the dominant energy sinks operating in the core of red giant stars are standard neutrinos and axions coupled with electrons. Any additional energy-loss process, not included in the stellar models, would reduce such a hint. Nevertheless, we find that values $g_{a e} / 10^{-13}>1.48$ can be excluded with $95 \%$ confidence.

Conclusions. The new bound we find represents the most stringent constraint for the axion-electron coupling available so far. The new scenario that emerges after this work represents a greater challenge for future experimental axion searches. In particular, we can exclude that the recent signal seen by the XENON1T experiment was due to solar axions.
\end{abstract}

Key words. elementary particles - stars: low-mass - globular clusters: general - Hertzsprung-Russell and C-M diagrams

\section{Introduction}

The observed properties of stars compared to model predictions have often been used to constrain various physical processes occurring in stellar interiors. In particular, the brightest stars on the red giant branch (RGB) of a globular cluster may probe some processes responsible for the energy generation, such as nuclear reactions, as well as the efficiency of energy sinks, the most important of which is the plasma neutrino emission. Indeed, in the core of these low-mass stars, which are close to the He ignition, the temperature reaches $\sim 100 \mathrm{MK}$, while the central density is $\sim 10^{6} \mathrm{~g} \mathrm{~cm}^{-3}$. According to the Standard Model, in this condition free electrons are highly degenerate and neutrinos are efficiently produced by plasma oscillations. Once produced, neutrinos escape from the stellar core, a process that subtracts the thermal energy spent for their production. On the other hand, degenerate electrons are excellent heat conductors and, in turn, they allow an efficient redistribution of the thermal energy. In addition, the high pressure of degenerate electrons hampers the core contraction and the consequent release of gravitational energy. As a result of the combined effects of neutrino production and electron degeneracy, the maximum temperature moves outward, to a layer placed approximately $0.2 M_{\odot}$ from the center, and the He ignition is delayed. In this framework, the 
luminosity of the RGB tip, which is the luminosity of a star at the off-center He ignition, is determined by the efficiency of these physical processes.

Although the scenario we describe here is widely accepted, additional energy sink processes, as predicted by the most popular extensions of the Standard Model, may greatly modify our understanding of the RGB evolution, and, in particular, they may affect the theoretical predictions of the RGB tip luminosity. Since the RGB tip luminosity is also considered a reliable standard candle, often used in cosmological studies (see, e.g., Freedman et al. 2020), it is important to investigate the possible impact of these additional energy-loss processes. Among them, the most appealing is probably the production of axions, or, more generally, of axion-like particles (ALPs). The existence of axions, which are small and weak interactive pseudo-scalar particles, was firstly proposed more than 40 years ago to solve the so-called strong CP problem, that is, the lack of evidence for a violation of the charge-parity symmetry by strong interactions (Peccei \& Quinn 1977; Weinberg 1978; Wilczek 1978). Furthermore, ALPs also emerge as pseudo-Nambu-Goldstone bosons of spontaneously broken global symmetries in high-energy extensions of the Standard Model.

Like plasma-neutrinos, axions or ALPs may be produced in the core of an RGB star by thermal processes, thus modifying the internal energy budget. The general rule is well known (Raffelt \& Weiss 1995): the larger the production rate of weak interactive particles produced by a thermal process, the brighter the tip of the RGB. For this reason, the RGB tip luminosity can be used to constrain the properties of neutrinos and other weak interactive slim particles (WISPs). In the case of axions, Bremsstrahlung should be the most efficient production mechanism in RGB stars, while other processes, like Primakoff and Compton, are suppressed because of the high electron degeneracy ${ }^{1}$.

In a previous attempt, Viaux et al. (2013a) made use of I-band photometric data of NGC 5904 (M5), a well-studied globular cluster of the Milky Way, to derive an upper bound for the strength of the axion-electron coupling $\left(g_{a e}\right)$. They found $g_{a e}<4.3 \times 10^{-13}$ (95\% C.L.). For this paper, we attempted to improve this constraint. Two are the major sources of uncertainty in the evaluation of the RGB tip luminosity: the bolometric corrections and the cluster distance. In addition to that, since the evolutionary timescale is rather short for stars in the brightest portion of the RGB, only few of them are usually observed within two or three magnitudes of the RGB tip. This occurrence hampers the clear identification of the RGB tip. In order to reduce this uncertainty, we extended the M5 constraint to other clusters. First of all, we considered $V$ and $I$ photometries of other two well-studied clusters, NCG 362 and NGC 104 (47 Tuc). In order to collect large and more complete samples of RGB stars, we combined catalogs of high spatial resolution Hubble Space Telescope (HST) images of the most crowded central regions with ground based observations of the external portions of the cluster fields. In addition, NGC 362 and NGC 104 are the only two galactic globular clusters (GGCs) for which a reliable distance can be estimated from the parallaxes reported in the latest Gaia Data Release 2 (Chen et al. 2018). Then, we extended our analysis to a sample of $22 \mathrm{GCs}$, for which near-infrared (near-IR) photometries are available following a wide observational campaign performed by Ferraro et al. (2000), Sollima et al. (2004), and Valenti et al. (2004a,b). The availability of JHK observations

\footnotetext{
1 In a recent paper (Straniero et al. 2019), we reviewed the axion production processes in stellar interiors and we provided the corresponding energy-loss rate to be used in stellar model calculations.
}

presents several advantages. Indeed, the brightest RGB stars are rather cool objects, and their spectral energy distribution is dominated by near-IR light. For this reason, near-IR bolometric corrections of RGB stars are more reliable than that required at shorter wavelengths, allowing a more reliable derivation of the bolometric magnitudes (see. e.g., Buzzoni et al. 2010). In addition, some of the brightest RGB stars are long period variables, whose amplitude may be quite large in the optical spectral range, while it is much smaller in the near-IR (Kiss \& Bedding 2003).

The models used to estimate the RGB tip luminosity of GGCs are presented in Sect. 2, while the adopted photometric samples and the derivation of the absolute bolometric magnitude of the RGB tip are described in Sect. 3. The statistical analysis and its results are discussed in Sect. 4. A summary and conclusions follow.

\section{Theoretical recipes}

\subsection{The RGB tip luminosity from stellar models}

The stellar models we use in the following analysis were computed by means of the Full Network Stellar evolution code (FuNS). This code has been extensively used to calculate models of stars of any mass and chemical composition and the related nucleosynthesis. We recently included the terms that account for the energy loss caused by an axion production possibly induced by various thermal processes in the energy conservation equation, in particular, Primakoff, Bremsstrahlung, $e^{+} e^{-}$pair annihilation, and Compton scattering on electrons or nuclei. The current version of the FuNS code and the relevant input physics are described in Straniero et al. (2019) ${ }^{2}$. It also contains a detailed description of the algorithms we use to calculate the axion energy-loss rates ${ }^{3}$. Here, we summarize the most important input physics adopted in the present work.

In general, the convective boundaries are fixed according to the Ledoux criterion. No convective overshoot is applied. In the convective zones, the temperature gradient is computed according to the mixing-length theory, as described in Cox \& Giuli (1968). The mixing length parameter, $\alpha=\Lambda / H_{P}=1.82$, was calibrated so as to reproduce the solar radius (Piersanti et al. 2007). The FuNS code may also account for stellar rotation (Piersanti et al. 2013) and microscopic diffusion (Straniero et al. 1997 and Piersanti et al. 2007), but these processes are not included in the present stellar models.

The FuNS has been optimized to handle large nuclear networks, such as those required to follow the neutron-capture nucleosynthesis in AGB stars (see Straniero et al. 2006). For the purpose of the present work, we considered 19 reactions for the $\mathrm{H}$ burning, those describing a full $\mathrm{pp}$-chain and $\mathrm{CNO}$-cycle, and an $\alpha$-chain for the He burning. All the stable isotopes of $\mathrm{H}$, $\mathrm{He}, \mathrm{Li}, \mathrm{Be}, \mathrm{C}, \mathrm{N}, \mathrm{O}$, plus ${ }^{20} \mathrm{Ne}$, are explicitly included in the nuclear network. The reaction rates are from version 6 of the STARLIB database (Sallaska et al. 2013). We note that most of the $\mathrm{H}$ and $\mathrm{He}$ burning reaction rates in this database are based on available experimental data. Rates of reactions for which no experimental information exists, or extrapolation to low/high temperatures are required, were obtained by means of statistical (Hauser-Feshbach) models of nuclear reactions (TALYS code; Goriely et al. 2008). The luminosity of the RGB tip is particularly sensitive to the adopted rates of the ${ }^{14} \mathrm{~N}(p, \gamma){ }^{15} \mathrm{O}$

\footnotetext{
2 See, in particular, Sect. 2 and the Appendix.

The same algorithms are also valid for the production of more general ALPs. Therefore, the conclusions of the present work apply to axions
} as well as to ALPs. 
O. Straniero et al.: The RGB tip of galactic globular clusters and the revision of the axion-electron coupling bound

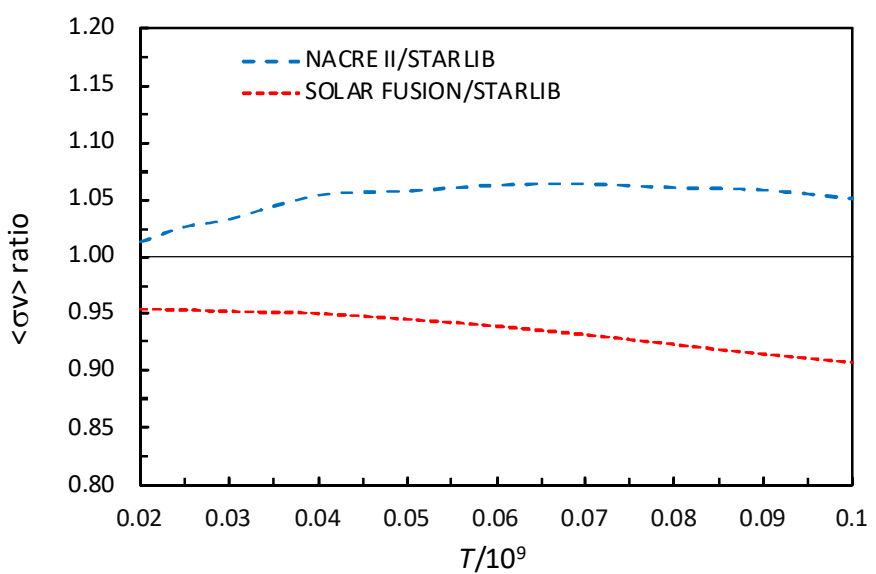

Fig. 1. ${ }^{14} \mathrm{~N}(p, \gamma){ }^{15} \mathrm{O}$ reaction rates suggested by the collaborations NACRE II and Solar Fusion are compared to the rate reported in the STARLIB repository.

and the triple- $\alpha$ reactions. The first reaction is the bottleneck of the CNO cycle, and, in turn, it determines the rate of growth of the core mass during the RGB phase. This reaction has been the object of an intense experimental campaign performed by the LUNA collaboration down to a center-of-mass energy of $70 \mathrm{keV}$, which corresponds to the Gamov peak for the typical temperature of the H-burning shell in evolved RGB stars (Formicola et al. 2004; Imbriani et al. 2004; Lemut et al. 2006). Our knowledge of this reaction is illustrated in Fig. 1, where the rate reported in the STARLIB repository (Sallaska et al. 2013), is compared to those reported in Adelberger et al. (2011) and in the NACREII repository (Xu et al. 2013). We note that in the temperature range of $20-100 \mathrm{MK}$, the differences are always smaller than $10 \%$. On the other hand, the triple- $\alpha$ reaction determines the He ignition, and, in turn, it fixes the brightest point attained by an RGB star. The most recent experimental investigation of this reaction was reported by Fynbo et al. (2005). Nevertheless, the widely adopted reaction rates, such as those reported in STARLIB or REACLIB (Cyburt et al. 2010), are all based on the NACRE rate (Angulo et al. 1999). In any case, in the temperature range of 100 to $300 \mathrm{MK}$, the differences between the Fynbo et al. (2005) and the NACRE rates are less than the quoted $20 \%$ error. Finally, screening corrections are computed according to Dewitt et al. (1973), Graboske et al. (1973), and Itoh et al. (1979), for weak, intermediate, and strong ion coupling, respectively. The importance of the nuclear screening in the computation of RGB models is discussed in Sect. 2.4.

Concerning the neutrino-energy-loss rates, the most important contribution in RGB stars is due to plasma neutrinos (Haft et al. 1994). FuNS also includes the energy losses due to photo and pair neutrinos (Itoh et al. 1996), Bremsstrahlung neutrinos (Dicus et al. 1976), and recombination neutrinos (Beaudet et al. 1967).

Radiative opacities are computed according to Lederer \& Aringer (2009), for $\log T \leq 4$, and to Iglesias \& Rogers (1996), at larger temperature, while electron conductivity are from Potekhin et al. (1999) and Potekhin (1999).

The equation of state $(\mathrm{EoS})$ is computed according to Rogers et al. (1996), for $\log T<6.5$, and to Straniero (1988) and Prada Moroni \& Straniero (2002), at larger temperature (see Sect. 2.3 for further details).

The Henyey method employed in the FuNS to solve the stellar structure equations is a first-order implicit method, and the accuracy of the solutions depends on the assumed mass and time resolutions. We employed an adaptive algorithm to select the
Table 1. Theoretical predictions of the tip bolometric magnitudes for globular cluster models with age $=13 \mathrm{Gyr}$ and $Y=0.25$.

\begin{tabular}{lllll}
\hline \hline$Z$ & {$[M / H]$} & $g_{13}$ & $M_{\text {bol }}$ & $\delta M_{\text {bol }}$ \\
\hline 0.0001 & -2.156 & 0.0 & -3.426 & 0.000 \\
0.0001 & -2.156 & 0.5 & -3.455 & 0.029 \\
0.0001 & -2.156 & 1.0 & -3.510 & 0.084 \\
0.0001 & -2.156 & 1.5 & -3.591 & 0.165 \\
0.0001 & -2.156 & 2.0 & -3.699 & 0.273 \\
0.0001 & -2.156 & 2.5 & -3.799 & 0.373 \\
0.0001 & -2.156 & 3.0 & -3.901 & 0.474 \\
0.0001 & -2.156 & 4.0 & -4.082 & 0.656 \\
0.0001 & -2.156 & 6.0 & -4.365 & 0.939 \\
\hline 0.001 & -1.156 & 0.0 & -3.643 & 0.000 \\
0.001 & -1.156 & 0.5 & -3.667 & 0.024 \\
0.001 & -1.156 & 1.0 & -3.715 & 0.072 \\
0.001 & -1.156 & 1.5 & -3.791 & 0.148 \\
0.001 & -1.156 & 2.0 & -3.881 & 0.237 \\
0.001 & -1.156 & 2.5 & -3.972 & 0.328 \\
0.001 & -1.156 & 3.0 & -4.062 & 0.419 \\
0.001 & -1.156 & 4.0 & -4.228 & 0.585 \\
0.001 & -1.156 & 6.0 & -4.513 & 0.870 \\
\hline 0.006 & -0.374 & 0.0 & -3.793 & 0.000 \\
0.006 & -0.374 & 0.5 & -3.815 & 0.022 \\
0.006 & -0.374 & 1.0 & -3.855 & 0.062 \\
0.006 & -0.374 & 1.5 & -3.915 & 0.123 \\
0.006 & -0.374 & 2.0 & -3.995 & 0.202 \\
0.006 & -0.374 & 2.5 & -4.074 & 0.281 \\
0.006 & -0.374 & 3.0 & -4.157 & 0.364 \\
0.006 & -0.374 & 4.0 & -4.315 & 0.522 \\
0.006 & -0.374 & 6.0 & -4.581 & 0.788 \\
\hline
\end{tabular}

Notes. From Cols. 1 to 5: metallicity (mass fraction), $[M / H]=$ $\log (Z / X)-\log (Z / X)_{\odot}$, coupling parameter $g_{13}=g_{a e} \times 10^{13}$, tip bolometric magnitude $M_{\text {bol }}=4.75-2.5 \log L / L_{\odot}$, difference with the tip bolometric magnitude of the $g_{13}=0$ model.

grid of mass shells and the time steps. In particular, the variations between adjacent mesh points of luminosity, pressure, temperature, mass, and radius should be:

$0.8 \times \delta<\frac{\left(A_{N+1}-A_{N-1}\right)}{A_{N}}<\delta$,

where $A$ is one of $L, P, T, M$, or $R$. In the present work, we used $\delta=0.05$ everywhere, except for the shells located around some critical points, such as the boundaries of the convective zones, for which we used $\delta=0.005$. Similarly, the algorithm that we used to fix the time steps limits the variations of physical and chemical variables. Typically, when models climb the RGB, the total number of mesh points is about 1500, while the time step decreases from $\Delta t \sim 3 \times 10^{4} \mathrm{yr}$, at $\log L / L_{\odot}=2.1$, down to $\Delta t \leq 10^{3} \mathrm{yr}$, for $\log L / L_{\odot}>3$. Finally, the external boundary conditions were fixed according to a scaled solar $T(\tau)$ relation (Krishna Swamy 1966).

The set of stellar models on which our analysis is based is illustrated in Table 1. These models share the age at the RGB tip, namely $\sim 13 \mathrm{Gyr}$, and the initial He mass fraction, namely $Y=0.25$. The metallicity $(Z)$ and the corresponding $[M / H]$ are listed in Cols. 1 and 2, respectively, while the resulting bolometric magnitudes at the RGB tip are reported in Col. 4. Variations of the cluster parameters, that is, age, $Y$, and $Z$ as well as the theoretical uncertainties are discussed in Sects. 3.5 and 2.3, respectively. 


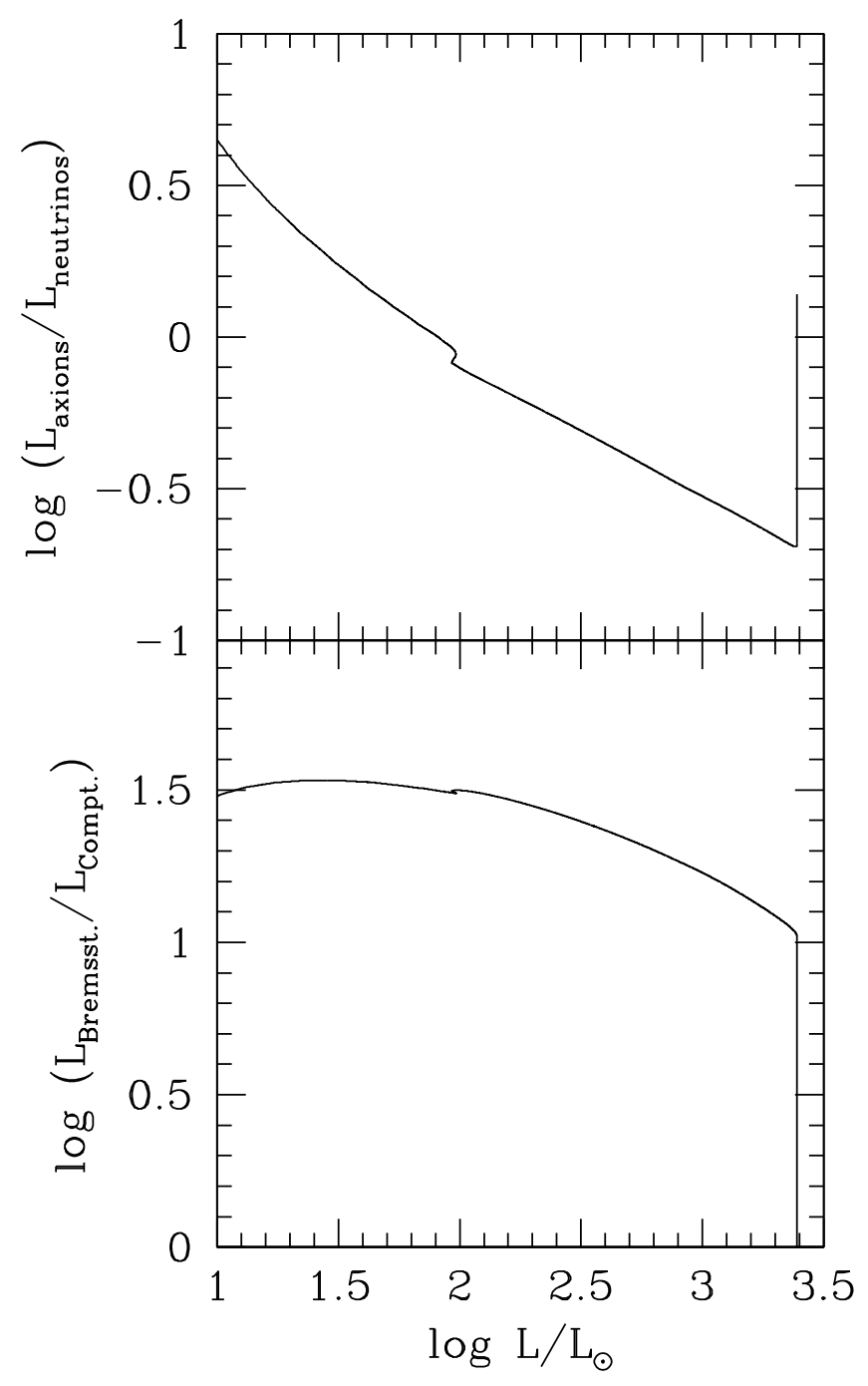

Fig. 2. Upper panel: evolution of ratio between axion and neutrino luminosites, from the base of the RGB $\left(\log L / L_{\odot}=1\right)$ to the RGB tip, for a model with $M=0.82 M_{\odot}, Y=0.25$, and $Z=0.001$. Only the coupling with electrons has been switched on $\left(g_{13}=1\right)$. Lower panel: evolution of the ratio between Bremsstrahlung and Compton axion luminosities.

\subsection{Axions by RGB stars}

In stellar interiors, axions can be produced through their interactions with standard model particles, mainly with photons and electrons, and, to a lesser extent, with nucleons. The Primakoff process, that is, the conversion of a photon into an axion in the electromagnetic field of an ion, $\gamma+Z e \rightarrow a+Z e$, is the major consequence of the coupling with photons. The energyloss rate due to this process depends on the square of $g_{a \gamma}$, a quantity representing the strength of the axion-photon coupling. This rate increases as $T^{4}$, but it is suppressed at the density of the RGB core due to the electron screening that reduces the effective charge of the nuclei. By adopting the present upper bound for the axion-photon coupling, that is, $g_{a \gamma}=6 \times 10^{-11} \mathrm{GeV}^{-1}$ (Ayala et al. 2014; Anastassopoulos et al. 2017), we found that the Primakoff process alone would produce a maximum decrease of the tip bolometric magnitude by $\sim 0.05 \mathrm{mag}$. Regarding the coupling with electrons, the most important axion sources at $T \sim 10^{8} \mathrm{~K}$ are the Compton scattering, $\gamma+e \rightarrow \gamma+e+a$, and the Bremsstrahlung, $e+Z e \rightarrow e+Z e+a$. As noted by Raffelt $\&$ Weiss (1995), owing to the large electron degeneracy, which develops

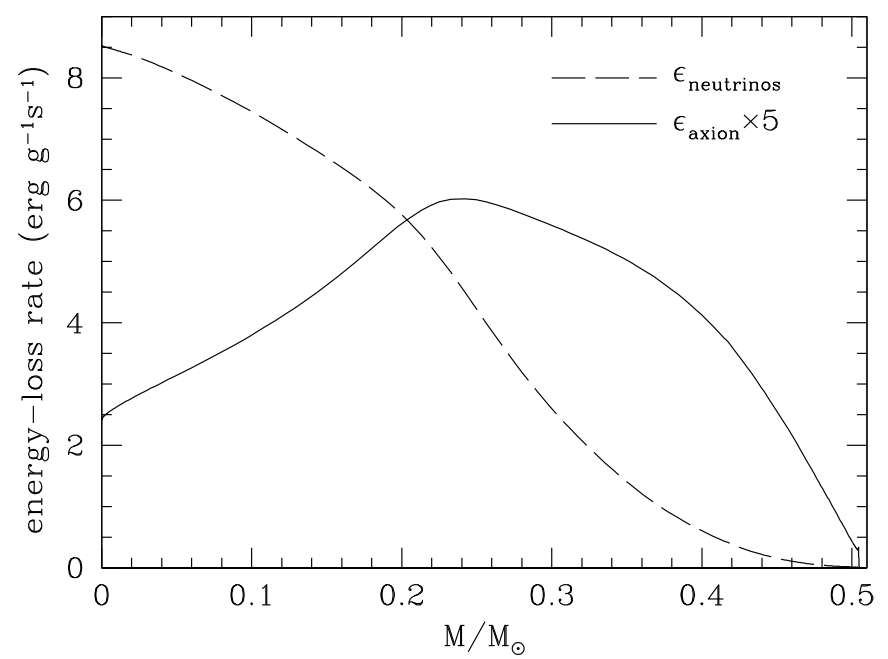

Fig. 3. Neutrinos and axions energy-loss rate within the core of a RGB model close to the RGB tip (just before the He ignition). Model parameters as in Fig. 2.

in the core of a red giant star, the Compton process is suppressed due to the Pauli blocking. On the contrary, the Bremsstrahlung process may induce a sizable axion production. The energy-loss rate due to Bremsstrahlung axion scales as the square of $g_{a e}$, a quantity representing the strength of the axion-electron coupling.

In Fig. 2, we compare the plasma-neutrino luminosity with those expected from axion emission due to Compton and Bremsstrahlung. This figure refers to a model with $M=$ $0.82 M_{\odot}, Y=0.25$, and $Z=0.001$, and an axion-electron coupling $g_{a e}=10^{-13}$. Almost the whole RGB evolution is shown, from $\log L / L_{\odot}=1$ to the tip. After the RGB bump, which corresponds to the double luminosity inversion occurring at $\log L / L_{\odot} \sim 2$, the neutrino emission becomes the dominant energy-loss process. Close to the RGB tip (just before the He ignition) the neutrino luminosity is about five times the axion luminosity. On the other hand, Bremsstrahlung is the leading axion-emission process in RGB stars, more than one order of magnitude brighter than Compton. Finally, Fig. 3 shows the energy-loss rates within the core just before the He ignition. We note that while neutrinos are mainly emitted near the center, where the density is higher, the axion energy loss peaks at $\sim 0.2$ $M_{\odot}$ out of the center, where the temperature attains its maximum.

The variations of the tip bolometric magnitudes as a function of $g_{a e}$ are reported in Table 1 for models with different initial metallicities (see also, Fig. 4). Here, $g_{13}=g_{a e} \times 10^{13}$. The corresponding reductions of the tip bolometric magnitudes with respect to models without axions are reported in the last column of Table 1 . We note that the more metal poor models show a higher sensitivity to the axion-electron coupling. As a whole, a $g_{13}=6$ would imply $\sim 1$ mag brighter bolometric magnitude, a value that is evidently incompatible with the observed RGB tip luminosity. A more stringent constraint can be obtained by means of a detailed evaluation of the error budget.

\subsection{Theoretical uncertainty}

For the purpose of the present work, we carried out a sensitivity study, with the aim of assigning a sort of theoretical error to the model prediction of the RGB tip luminosity. There are many examples of this kind of study in the extant literature (Viaux et al. 2013b; Valle et al. 2013; Serenelli et al. 2017). In these 


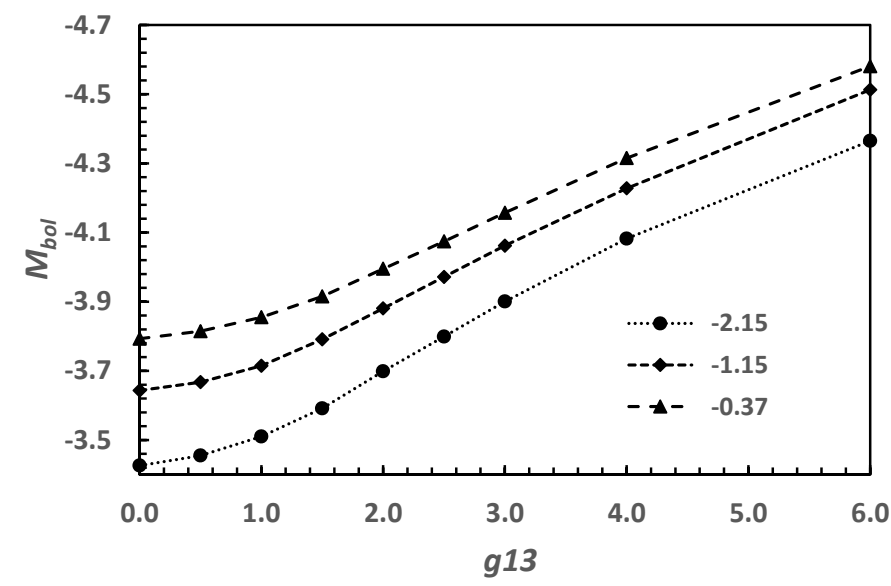

Fig. 4. Relation $M_{\text {bol }}$ at the RGB tip versus $g_{13}$ for 3 different metallicities $([M / H])$.

papers, the reader can find exhaustive illustrations of the various uncertainties affecting the relevant input physics. We do not repeat this discussion here. The uncertainties we adopted are listed in Table 2. From Col. 1 to Col. 4, we report: (1) the model inputs; (2) the reference for the preferred values; (3) the assumed $1 \sigma$ errors; (4) the variations of the RGB tip bolometric magnitudes implied by the variation of each input physics within the assumed error bar (full width). The quantities $\Delta M_{\mathrm{bol}}$ reported in the last column represent the sensitivity of the predicted tip bolometric magnitude to the various model inputs. We note that the relationship between tip luminosity and model input is often nonlinear, so the uncertainty may be not perfectly symmetric around the central $M_{\text {bol }}$ value. For this reason, in the last column of Table 2 we report the full-width variation rather than the half-width one. In any case, a rough estimation of the cumulative theoretical error may be obtained by summing the (average) half-width variations in quadrature, that is, $\Delta M_{\mathrm{bol}} / 2$. The result is $\sigma_{\text {theo }} \sim 0.04$. On the other hand, a more accurate estimation of this error can be obtained by means of a Monte Carlo propagation method. In practice, we assumed that each one of the listed model inputs (except for the microscopic diffusion and EoS) may vary according to a normal error distribution function. This assumption, which is appropriate when the uncertainty represents statistical fluctuations of experimental measurements, is certainly not strictly appropriate when the model input has been obtained through a theoretical calculation. Nevertheless, this is still a reasonable and simple assumption when the theory can identify a preferred (or best) value for the model input and when values far from it are less likely than values close to it. For example, the neutrino energy-loss rates are computed on the basis of the Weinberg-Salam electro-weak theory (see, e.g., Raffelt 1996). In the last 30 years, precision experiments have tested this quantum field theory at the level of one percent or better. Nevertheless the neutrino rates depend on some constants, such as the Weinberg angle, not predicted by the theory, whose values are determined experimentally. This occurrence introduces an uncertainty that we assume to be modeled by means of a proper normal error distribution ${ }^{4}$.

On the other hand, such an approach cannot be applied when the theory can not identify a preferred (or best) value of the model input, but a range of equally possible values. In that case

\footnotetext{
4 The possible existence of a nonzero neutrino magnetic moment, which would imply systematic increases of the energy-loss rates, is not considered here (see Sect. 5).
}

a rectangular probability distribution is more appropriate. This is the case of microscopic diffusion and EoS.

The term EoS should be intended as a set of relations we used to calculate all the thermodynamical quantities that appear in the stellar structure equations. It includes the density, the specific heats, the adiabatic gradient, and the partial derivatives of the density with respect to pressure, temperature, and molecular weight. We note that in FuNS we used $P$ and $T$ as native thermodynamic variables. For $\log T<6.5$, we used the latest version of the OPAL EoS, while for larger temperatures we adopted the EoS described in Straniero (1988) and Prada Moroni \& Straniero (2002). The latter assumes fully ionized matter and includes a detailed description of the relevant quantum-relativistic effects as well as the ion and electron Coulomb coupling. No approximate formulas were used for the evaluation of the Fermi-Dirac integral, which are always computed numerically. On the other hand, the OPAL EoS represents the state-of-the-art for the computation of the thermodynamical quantities in the partially ionized H-rich envelope. As a whole, the most important uncertainties affecting EoS calculations concern the deviations from a perfect gas that arise at high density. Then, to evaluate the sensitivity of the RGB tip luminosity on the EoS, we computed some stellar models by assuming a perfect gas EoS. Firstly, we removed all the Coulomb corrections in the EoS calculation for $\log T>6.5$. These corrections should mainly affect the core of the brightest RGB models, where the central density approaches $\sim 10^{6} \mathrm{~g} \mathrm{~cm}^{-3}$. In this case, the resulting tip bolometric magnitude is 0.0025 mag larger than that obtained with the reference models. Then, we have replaced the OPAL EoS with a perfect gas low-temperature EoS, in which the ionization degree was obtained with a classical Saha equation. In this case, the resulting tip bolometric magnitude differs by less than 0.001 mag with respect to the reference case. Hence, we assumed a conservative systematic uncertainty associated with the EoS of 0.003 mag.

Concerning microscopic diffusion, the effect of the combined actions of gravitational settling and thermal diffusion in main sequence stars is that $\mathrm{He}$ and heavy elements are slowly moved toward the stellar center. In the FuNS code, microscopic diffusion is taken into account by inverting the set of Burgers equations (Thoul et al. 1994). We note that in the evaluation of the Coulomb logarithm, full ionization is often assumed. Although this approximation is very common among the extant calculations of evolutionary tracks and isochrones for globular cluster stars, it presents some limitations. For $\log T<6$, the Coulomb cross-section is overestimated when full ionization is assumed, and, in turn, the diffusion coefficients are underestimated. In addition, the gravitational settling may be hampered by the acceleration caused by the net transfer of momentum from the outgoing photon flux to ions and electrons, a process also known as radiative levitation (Turcotte et al. 1998; Delahaye \& Pinsonneault 2005, and references therein). We note that the interior of a main-sequence star with mass $\sim 0.8 M_{\odot}$ is mostly stable against convection, with the exception of a small external convective zone, so that microscopic diffusion is the major instability affecting the internal composition. Owing to the large main sequence lifetime, this secular instability may produce sizeable modifications to the chemical stratification, and, in turn, of the physical structure (Proffitt \& Vandenberg 1991; Chaboyer et al. 1992, and Straniero et al. 1997). However, just after the central $\mathrm{H}$ exhaustion, the convective instability penetrates inward, so about $80 \%$ of the stellar mass is homogenized (first dredge up). As a consequence, the original amounts of He and heavy elements are restored in a large part of the star, and the evolutionary track resembles that of a model 
Table 2. Uncertainties of the input physics adopted in the calculations of the tip bolometric magnitudes.

\begin{tabular}{lccc}
\hline \hline Parameter & Reference & Uncertainty & $\Delta M_{\text {bol }}(a)$ \\
\hline$\eta$ (mass loss) & Reimers & $0.1: 0.5$ & 0.014 \\
$14 \mathrm{~N}+\mathrm{p}$ & STARLIB (LUNA) & $\pm 10 \%$ & 0.016 \\
$3 \alpha$ & STARLIB (NACRE) & $\pm 20 \%$ & 0.033 \\
Screening $3 \alpha$ & Dewitt et al. (1973) + Graboske et al. (1973) + Itoh et al. (1979) & $\pm 20 \%$ & 0.035 \\
Neutrinos & Haft et al. (1994) + Itoh et al. (1996) & $\pm 5 \%$ & 0.026 \\
$e$ conductivity & Potekhin et al. (1999) & $\pm 5 \%$ & 0.049 \\
rad. opacity & OPAL+COMA 2006 & $\pm 5 \%$ & $<0.001$ \\
$\alpha$ (mix. length) & 1.82 (SSM calibrated) & $1.62: 2.02$ & $<0.001$ \\
Boundary condition & $T(\tau)$ rel. (Krishna Swamy 1966) & $\pm 10 \%$ & $<0.001$ \\
Eq. of state & OPAL 2005 + Straniero (1988) & See text & 0.003 \\
Microscopic diffusion & Thoul et al. (1994) & See text & $0.006-0.025$ \\
\hline
\end{tabular}

Notes. ${ }^{(a)}$ Full width variation of the tip bolometric magnitude.

computed without diffusion. Later on, during the RGB phase, the evolutionary lifetime becomes much shorter than the diffusion timescale. Nonetheless, the star still keeps some memory of the modifications induced by diffusion in the previous evolutionary phase. Small changes in some critical quantities are found. In particular, RGB models with diffusion present a larger core mass at the He flash and, in turn, a higher RGB tip luminosity.

In our reference models (Table 1), we switched off the microscopic diffusion. Nonetheless, in our analysis we considered its effect as a systematic uncertainty. In practice, to maximize the effects of microscopic diffusion, we neglected the radiative levitation. As a result, the tip bolometric magnitudes are from $-0.006 \mathrm{mag}$ (at $Z=0.006$ ) up to $-0.025 \mathrm{mag}$ (at $Z=0.0001$ ) brighter than that of the corresponding reference models (no diffusion).

In summary, in the Monte Carlo calculation we assumed that the uncertainties associated with the EoS and microscopic diffusion produce equally probable variations in between the two extreme cases previously described. For all the other model inputs, we assumed a normal error distribution with $\sigma$ values as reported in Col. 3 of Table 2. Some targeted checks have been done to verify that the variations of the tip luminosity implied by the variations of the various model inputs are uncorrelated. This occurrence allows us to evaluate the cumulative shift of the tip luminosity simply by summing the independent effects induced by the variations of the single model inputs. Then, we calculated a large number $\left(10^{5}\right)$ of tip $M_{\text {bol }}$ by randomly varying the model inputs. The result for an hypothetical Cluster with age $=13 \mathrm{Gyr}$, $[M / H]=-1$ and $Y=0.25$ is illustrated in Fig. 5. The normalized frequency histogram is well fit by a Gaussian distribution with mean $\Delta M_{\text {bol }} \sim 0$ and standard deviation STD $=0.038$. Finally, we repeated the Monte Carlo calculation, changing the cluster parameters, that is, the age and the initial composition, finding only marginal variations in the resulting standard deviation. In summary, the adopted theoretical error of the tip bolometric magnitude is $\sigma_{\text {theo }}=0.038$.

\subsection{Comparisons with previous calculations}

In the extant literature there are several papers reporting calculations of RGB stellar models for globular cluster stars. Here, we compare our results with the most recent ones, as obtained with four different evolutionary codes, namely BaSTI (Pietrinferni et al. 2004) $)^{5}$, PGPUC (Valcarce et al. 2012),

\footnotetext{
5 http://basti.oa-teramo.inaf.it
}

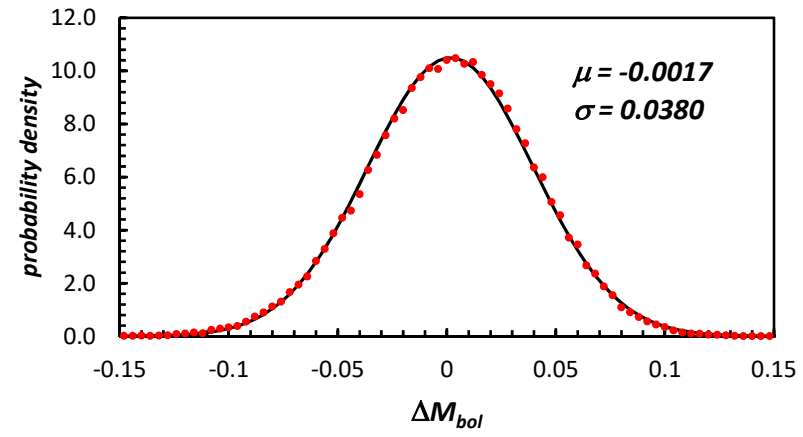

Fig. 5. Result of Monte Carlo error propagation: points represent the normalized frequency of Monte Carlo events, while the curve is the best-fit Gauss. function. The mean and standard deviation are shown.

GARSTEC (Serenelli et al. 2017), and PISA (Dell'Omodarme et al. 2012) ${ }^{6}$. To perform these comparisons, we computed specific models with the same initial stellar parameters as in the previous calculations. The results of these comparisons are reported in Table 3. In general, the differences in the tip bolometric magnitude are within the quoted $1 \sigma$ theoretical uncertainty (see Sect. 2.3). However, a larger discrepancy is found with respect to the PGPUC model, the latter being about 0.09 mag fainter (or $\left.\delta \log L / L_{\odot}=0.037\right)$. Since this model is the same one adopted by Viaux et al. (2013a) to constrain the axion-electron coupling, we attempted to understand the origin of this discrepancy. This faintness of the PGPUC models at the RGB tip was already noted by Serenelli et al. (2017). These authors suggested that it is due to the evaluation of the screening enhancement factors that multiply the nuclear reaction rates. Indeed, Viaux et al. (2013a,b) followed the prescriptions of Salpeter (1954), who developed the theoretical framework for the calculation of the screening potential in the two extreme cases of weak and strong regime of the stellar plasma. In the weak screening regime, the coupling between a nucleus and the nearby electrons and nuclei is weak, or, more precisely, the Coulomb interaction energy is much smaller than the thermal energy $(K T)$. On the other hand, the strong regime refers to the case of strong coupling, that is, Coulomb interaction energy much greater than the thermal energy. The weak regime condition is usually fulfilled in the core of H-burning stars with masses $\sim 0.8 M_{\odot}$. However, at the higher

\footnotetext{
6 http://astro.df.unipi.it/stellar-models
} 
Table 3. Comparisons with previous calculations.

\begin{tabular}{lcccccccc}
\hline \hline$M / M_{\odot}$ & $Y$ & $Z$ & $\log L / L_{\odot}($ FuNS $)$ & BASTI & PGPUC & GARSTEC & PISA & Weak sc (FuNS) \\
\hline 0.82 & 0.245 & $1.36 \mathrm{E}-03$ & 3.337 & & -0.037 & & & -0.030 \\
0.80 & 0.246 & $1.00 \mathrm{E}-03$ & 3.358 & 0.015 & & -0.022 & -0.009 & \\
0.80 & 0.245 & $1.00 \mathrm{E}-04$ & 3.272 & 0.020 & & -0.022 & -0.005 & \\
\hline
\end{tabular}

Notes. The model parameters, that is, mass, metallicity, and He mass fraction are reported in the first three columns. The RGB tip luminosity obtained with the FuNS code is in Col. 4, while the corresponding differences with respect previous calculations are in Cols. 5-8. The difference with respect to the FuNS calculation, as obtained imposing the weak nuclear screening everywhere and at any time (see text), is in the last column.

density of the core of an RGB star $\left(\sim 10^{6} \mathrm{~g} \mathrm{~cm}^{-3}\right)$, the interaction energy is comparable to the thermal energy, and the weak screening prescription by Salpeter (1954) largely overestimates the screening factors. As shown by Dewitt et al. (1973) and Graboske et al. (1973), the intermediate regime is more appropriate to describe the plasma conditions in the core of an RGB star. As an example, for a He-rich gas of which the temperature and density are $10^{8} \mathrm{MK}$ and $10^{6} \mathrm{~g} \mathrm{~cm}^{-3}$, respectively, the effective $3 \alpha$ rate is more then a factor of 2 larger when the weak screening is imposed. As a result, the He ignition should occur at lower luminosity. To quantify this effect, we calculated an additional model, $M=0.82 M_{\odot}, Z=0.00136$, and $Y=0.25$, by imposing week screening factors for all the nuclear reactions, from the beginning of the evolution up to the RGB tip. As reported in the last column of Table 3, at the RGB tip this model is about 0.075 mag fainter than our reference model computed by calculating the screening factors according to Dewitt et al. (1973) and Graboske et al. (1973). We conclude that most of the discrepancies between our models and the PGPUC ones are likely due to the different nuclear screening prescriptions. As we show in Sect. 4, the larger RGB tip luminosity we obtain by adopting the intermediate screening reduces the need for additional energy loss from the RGB core, and, in turn, it implies a lower upper bound for $g_{a e}$.

Finally, we wish to mention a discrepancy between our sensitivity study and that discussed in Valle et al. (2013). At variance with our result, these authors find that the major uncertainty affecting the RGB tip luminosity is due to a variation of the radiative opacity. On the other hand, our result confirms previous findings reported by Viaux et al. (2013a) and Serenelli et al. (2017). In principle, the energy transport within the core of an RGB star is mainly driven by the heat conduction from degenerate electrons, so that a $5 \%$ variation of the radiative opacity at high temperature is expected to produce a small effect on the tip luminosity. Outside the core, the radiative opacity affects the temperature gradient, which is super-adiabatic in a large portion of the convective envelope. As a result, the effective temperature of an RGB star is modified by a change of the radiative opacity, but its luminosity is only marginally affected. For these reasons, although further investigations are certainly needed to clarify the origin of such a discrepancy, we are quite confident about the robustness of our prediction.

\section{Combining HST photometries with those from ground-based telescopes}

In this section, we illustrate the strategy we followed to derive the RGB tip bolometric magnitudes from optical ( $V$ and $I$ ) and near-infrared $(J$ and $K$ ) photometries.

\subsection{The VI catalog}

In order to select the most complete samples of RGB stars, the adopted strategy was to combine different catalogs characterized by complementary properties. In particular, to resolve the crowded central regions, we exploited the high spatial resolution of space-based telescopes, while to sample the entire radial extension of the clusters, we used photometric data from groundbased facilities that cover a larger field of view (FoV). To this aim, the required data sets were extracted from public catalogs, namely the ACS globular cluster survey (Sarajedini et al. 2007) and the homogeneous UBVRI photometry of globular clusters (Stetson et al. 2019). In particular, we used $V$ and $I$ magnitudes from the latter and the magnitudes in the filters F606W and F814W, as calibrated into the ground photometric system, for the former. By using common stars, we carefully checked that the ground system magnitudes tabulated into the HST catalog and those from the ground-based catalogs were homogeneous.

For the present work, we selected three clusters, namely M5, NGC 362 and 47 Tuc. The first is the cluster already used by Viaux et al. (2013b) to constrain the strength of the axionelectron coupling, while the other two clusters are the only ones for which reliable parallax distances are available after the Gaia DR2 (Chen et al. 2018). In the specific case of NGC 362 , since the ground-based catalog was not complete as for M5 and 47 Tuc, we have complemented our analysis with additional observations from the ESO archive, that is, FORS2 data from the program 60.A-9203. In particular, we analyzed two short images $(1 \mathrm{~s}$ each) obtained with the standard resolution $\left(0^{\prime \prime} .25 \mathrm{pixel}^{-1}\right.$ for a total FoV of $6^{\prime} .8 \times 6^{\prime} .8$; hereafter SR $)$ and two $\left(1 \mathrm{~s}\right.$ each) with a high-resolution $\left(0^{\prime \prime} .125\right.$ pixel $^{-1}$ for a total FoV of $4^{\prime} .2 \times 4^{\prime} .2$; hereafter HR) collimator in both $I_{\mathrm{BESS}}$ and $V_{\mathrm{BESS}}$ filters. For both data sets (i.e., HR and SR), the photometric analysis was performed using DAOPHOT II (Stetson 1987). Briefly, for each exposure, we first modeled a spatially variable point spread function using more than 50 isolated and bright stars. We then applied this model to all the sources detected more than $3 \sigma$ from the background level, and we built the final catalog containing all the sources detected in both filters. Finally, we report the instrumental magnitudes to the absolute reference system by cross-correlation ${ }^{7}$ with the standard Ste-tson catalog. Then, the combined catalogs were built by joining together the different available catalogs. When possible, we preferentially adopted the HST magnitude, which is not prone to crowding. However, for a few central very bright stars, we nevertheless preferred to adopt the ground based magnitudes, since the HST magnitudes may be

7 To perform the cross-correlation, we used CataXcorr, a code aimed at cross-correlating catalogs and finding astrometric solutions, developed by P. Montegriffo at INAF - OAS Bologna. This package has been successfully used in a large number of papers in the past years, in particular, Pallanca et al. (2013, 2014, 2019), Cadelano et al. (2017), and Dalessandro et al. (2014). 
biased because of the nonlinear regime close to the photometric saturation level. We note that for such bright objects, the crowding is not a problem, even in the most central regions. In the case of NGC 362, for which two FORS2 data sets were also available, the priority adopted in the catalog compilation was HST, FORS2 HR, FORS2 SR, and Stetson standard, except for very bright objects, for which we adopted the FORS HR magnitudes even if detected in the HST catalog. Finally, we dereddened the magnitudes by adopting the $E(B-V)$ values tabulated in Harris (1996, 2010 version) and a standard extinction law with $R v=3.1$. Following Cardelli et al. (1989) and O'Donnell (1994), we assumed $A_{V}=3.12 E(B-V)$ and $A_{I}=1.87 E(B-V)$.

The apparent bolometric magnitudes of the RGB stars were calculated by means of the UBVRIJHK color-temperature calibration reported in Worthey \& Lee (2011). In all cases, we assumed $\log g=0$, which is a typical gravity value for bright RGB stars. Then, we estimated the bolometric correction of all stars making use of their own dereddened color $(V-I)_{0}$. The combined errors on the bolometric magnitudes were calculated as the propagation of several sources of uncertainty: namely the photometric error as estimated with DAOPHOT, all the sources of errors in the determination of the calibrated and dereddened magnitudes (e.g., the value of reddening $E(B-V)$, the extinction law, the calibration zero points, etc.), and the error on the bolometric correction. The latter includes the intrinsic uncertainty of the BC-color empirical relation by Worthey \& Lee (2011) and that of the measured $V-I$ colors. For the brightest RGB stars, this uncertainty is of the order of \pm 0.15 mag and it represents the major contribution to the $m_{\mathrm{BOL}}$ error budget.

\subsection{The near-IR catalog}

In addition to the $V I$ photometry described in the previous section, we also considered the near-IR catalog of GGCs presented in a series of papers by the Bologna team (Ferraro et al. 2000; Valenti et al. 2004a). The catalog contains a set of JHK photometric data of 22 GGCs, obtained at the ESO MPI $2.2 \mathrm{~m}$ telescope equipped with the near-IR camera IRAC-2. These data cover the most central $4 \times 4 \operatorname{arcmin}^{2}$ regions of each cluster. In some cases, additional data from the 2 mass survey have been used to sample more external regions (Valenti et al. 2004a). For five clusters, that is, M3, M5, M10, M13, and M92, we also considered the $J$ and $K$ observations obtained by Valenti et al. (2004b) with the Telescopio Nazionale Galileo (TNG) equipped with the ARNICA IR camera. These observations cover the most crowded $1.5 \times 1.5 \operatorname{arcmin}^{2}$ central regions. Finally, a rather large sample of giants belonging to the RGBa of $\omega \mathrm{Cen}$, as obtained by Sollima et al. (2004) with the ESO NTT telescope during the commissioning of the SOFI camera, was also used. This data set covers a $13 \times 13 \operatorname{arcmin}^{2}$ area around the cluster center. Then, the bolometric magnitudes were estimated by means of the empirical bolometric corrections derived by Buzzoni et al. (2010). In particular, we used their $\mathrm{BC}_{K}$ versus $(J-K)$ color relation. As for the $V I$ photometriic samples, the errors of the bolometric magnitudes were calculated by combining the uncertainties due to the photometry with those affecting the bolometric correction. In general, the $K$-band bolometric corrections are more reliable than the $V$ and $I$ ones. Following Buzzoni et al. (2010), for the brightest RGB stars, we assumed $\sigma_{\mathrm{BC}}= \pm 0.1 \mathrm{mag}$.

\subsection{Selection of RGB stars}

Particular attention has been paid to decontaminate the RGB photometric samples from background field stars and from the

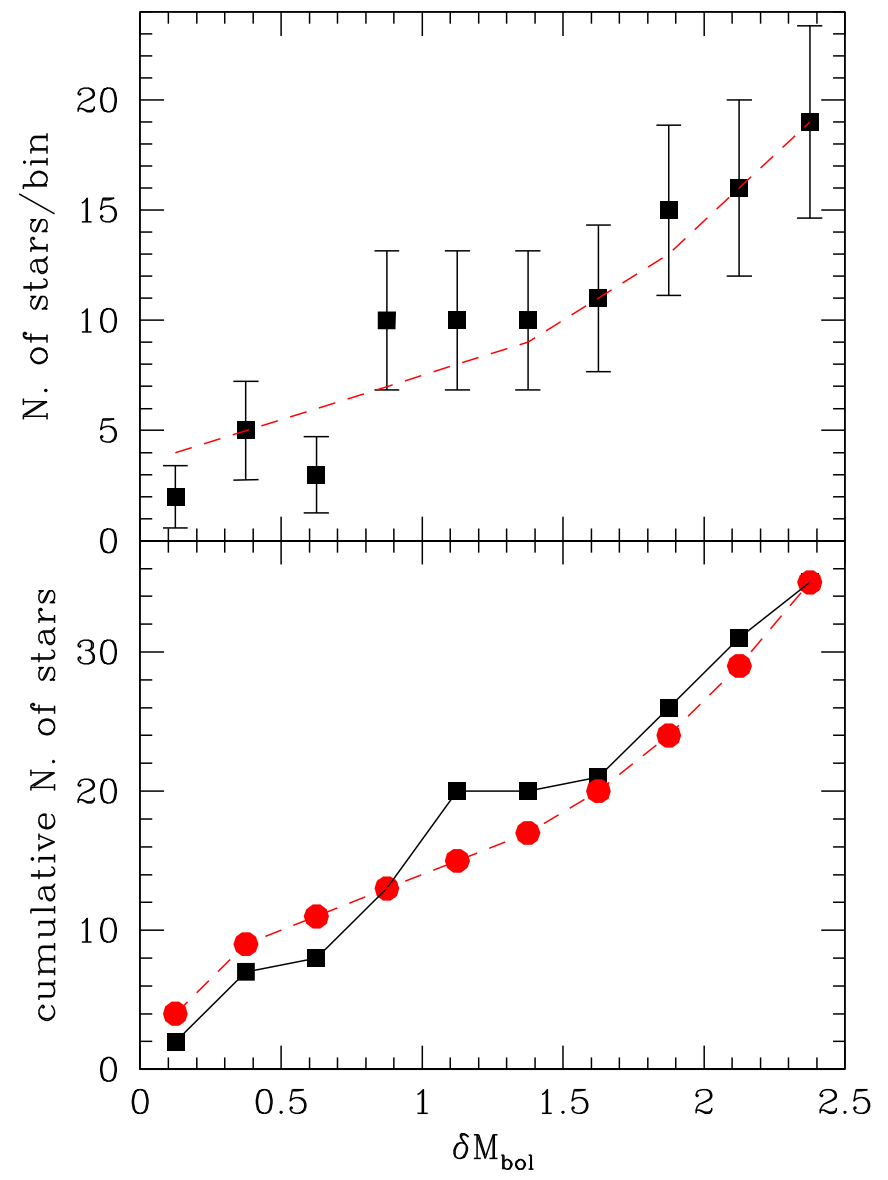

Fig. 6. Upper panel: observed luminosity function of the 2.5 mag brightest portion of the RGB of cluster M5 (squares) is compared to the theoretical luminosity function (dashed line). Poisson errors $(\sqrt{N})$ are also shown. Lower panel: the cumulative LF of the brightest portion of the M5 RGB (solid line and squares) is compared to the theoretical expectation (dashed line and circles).

presence of AGB stars. The selection of RGB star candidates was done based on the available colors and magnitudes (VIJHK). Given the very small photometric errors of RGB and AGB stars ( $\sim 0.01$ mags) and the expected color separation between the two parent sequences $\left(0.02<(V-I)_{\mathrm{RGB}}-(V-I)_{\mathrm{AGB}}<0.1\right)$, we found a clear separation between the two populations from a minimum of $2 \sigma$, around 1 mag below the RGB-tip, up to more than $20 \sigma$ at larger magnitudes. A good example of this RGB-AGB separation can be found in Fig. 1 of Beccari et al. (2006), where the two populations of 47 Tuc, thanks to the high quality of the data similar to that of the present work, are well populated and clearly distinguishable. Such a clear separation allowed us to select the RGB sample through a box selection drawn around the RGB fiducial line and properly checked by a visual inspection.

\subsection{The RGB tip luminosity from GGC photometries}

In this section, we illustrate the method we followed to determine the luminosity of the RGB tip from a finite photometric sample of RGB stars. This problem is equivalent to the evaluation of the difference of the luminosity of the RGB tip and that of the brightest observed RGB star. To do that, we made use of theoretical luminosity functions (LFs) and synthetic colormagnitude diagrams (SCMDs). First of all, we verified that the observed and the theoretical LFs were drawn from the same 
O. Straniero et al.: The RGB tip of galactic globular clusters and the revision of the axion-electron coupling bound

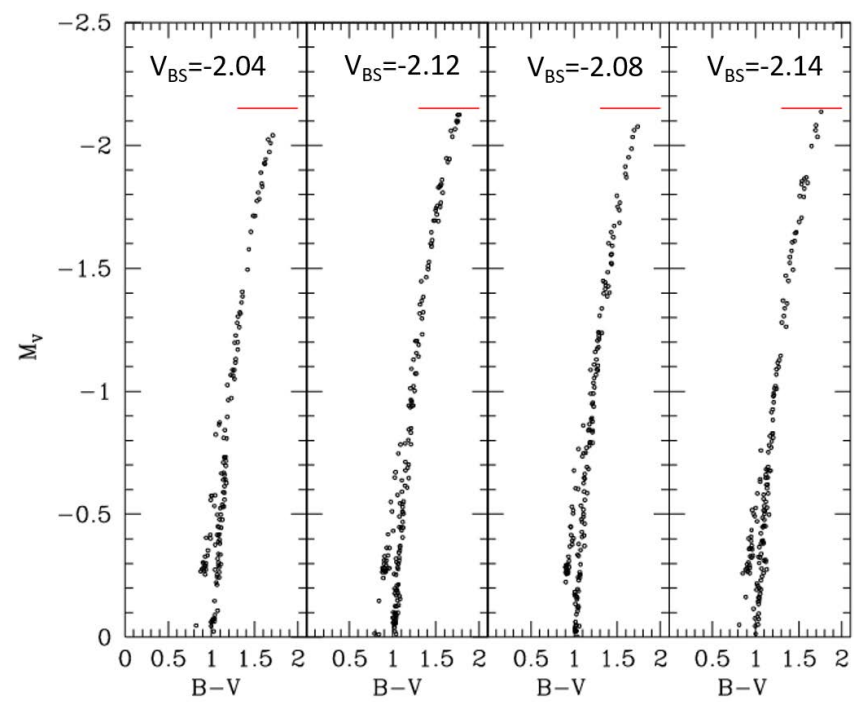

Fig. 7. Synthetic color-magnitude diagrams of bright globular cluster stars. All SCMDs were computed with the same input parameters; in particular, the same age, chemical composition, and number of stars. The location of the RGB tip is marked by a red horizontal line. For all the SCMDs, it is at $M_{V}=-2.15$. The $M_{V}$ mag of the brightest stars are reported in each panel $\left(V_{\mathrm{BS}}\right)$.

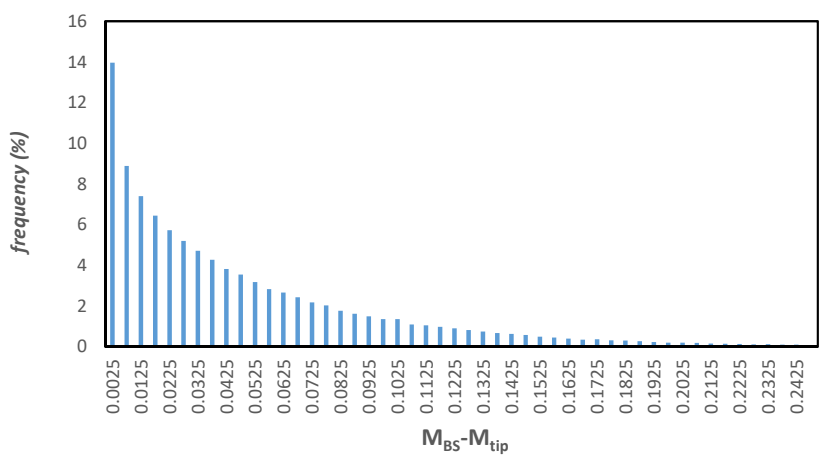

Fig. 8. Histogram representing frequency of differences between the bolometric magnitute of the brightest star $\left(M_{\mathrm{BS}}\right)$ and that of the RGB tip $\left(M_{\text {tip }}\right)$ for a sample of $10^{5}$ SCMDs computed for an age of $13 \mathrm{Gyr}$, $Z=0.001, Y=0.25$ and with 100 stars within $2.5 \mathrm{mag}$ from the RGB tip.

distribution. In Fig. 6, the observed luminosity function of the cluster M5 is compared to the theoretical expectation. By means of a classical Kolmogorov-Smirnov test, we found a statistic parameter $d=0.2$ and a probability of $97.5 \%$ that the theoretical and the observed data sets are drawn from the same distribution. Furthermore, a chi-squared test leads to a $86 \%$ probability that the theoretical and the observed LFs are drawn from the same distribution. These statistical tests support our use of the theoretical LFs to evaluate the differences between observed luminosity of the brightest RGB stars and the RGB tip luminosity. Then, synthetic color-magnitude diagrams were generated following a standard Monte Carlo procedure. Some examples are illustrated in Fig. 7. In computing these four SCMDs, we assumed the same input parameters; in particular, the same age, chemical composition, $B$ and $V$ photometric uncertainties, and total number of stars. In all cases, the RGB tip occurs at $M_{V}=-2.15 \mathrm{mag}$. In contrast, the location of the brightest star changes randomly because of statistical fluctuations.

The analysis of these fluctuations can be done by collecting a sufficient number of these SCMDs, as illustrated in Fig. 8.
Table 4. Medians, standard deviations, and mean deviations from the median of the corrections applied to the bolometric magnitude of the brightest RGB stars.

\begin{tabular}{lcccccc}
\hline \hline Age (Gyr) & $Z$ & $Y$ & $N^{(a)}$ & Median & STD & MDM \\
\hline 13 & 0.001 & 0.25 & 50 & 0.063 & 0.107 & 0.072 \\
13 & 0.001 & 0.25 & 100 & 0.030 & 0.056 & 0.035 \\
13 & 0.001 & 0.25 & 200 & 0.008 & 0.030 & 0.018 \\
13 & 0.001 & 0.24 & 100 & 0.030 & 0.056 & 0.035 \\
13 & 0.0001 & 0.25 & 100 & 0.028 & 0.063 & 0.037 \\
11 & 0.001 & 0.25 & 100 & 0.030 & 0.055 & 0.035 \\
\hline
\end{tabular}

Notes. ${ }^{(a)}$ Number of stars in the brightest 2.5 mag of the RGB.

This frequency histogram represents the distribution of the differences between the bolometric magnitude of the brightest stars and that of the RGB tip in a sample of $10^{5}$ SCMDs. Each SCMD was computed assuming an age of $13 \mathrm{Gyr}, Z=0.001$, and $Y=$ 0.25 . In addition, each SCMD contains 100 stars in the brightest 2.5 mag portion of the RGB. The mode of the probability distribution is evidently 0 , while the median is $\langle\delta\rangle_{100}=0.030 \mathrm{mag}$, and the standard deviation from the median is STD $=0.056 \mathrm{mag}$. However, for probability distributions with a long right tail, the standard deviation overestimates the dispersion. For this reason, the mean deviation from the median $\left(\mathrm{MDM}^{8}\right)$, or the interquartile range, were often preferred to the STD. For the probability distribution shown in Fig. 8, we find $\mathrm{MDM}_{100}=0.035 \mathrm{mag}$, while the first and the third quartiles are at -0.019 and +0.033 with respect to the median. We note that the semi-interquartile range is $0.026 \mathrm{mag}$, which is slightly smaller than MDM and about half the STD. In Table 4, we report the values of medians, standard deviations, and mean deviations from medians, as obtained under different assumptions about age, chemical composition, and the parameter $N$ that represents the number of stars in the brightest portion of the RGB. It results that the statistical descriptors are practically insensitive to variations of age, $Y$, and $Z$, within the range expected for globular cluster stars. In contrast, they are substantially affected by the number of bright RGB stars. Then, knowing the bolometric magnitude of the brightest RGB star $\left(m_{\mathrm{BS}}\right)$ and the number of stars in the brightest $2.5 \mathrm{mag}$ of the observed RGB $(N)$ for each cluster, we can estimate the tip bolometric magnitude as: $m_{\text {tip }}=m_{\mathrm{BS}}-\langle\delta\rangle_{N}$, where $\langle\delta\rangle_{N}$ is the median of the distribution function of $m_{\mathrm{BS}}-m_{\text {tip }}$. Concerning the total error, we assumed $\sigma_{\text {tip }}^{2}=\sigma_{\mathrm{BS}}^{2}+\mathrm{MDM}^{2}$, where $\sigma_{\mathrm{BS}}$ is the error on the apparent bolometric magnitude of the brightest RGB star (see Sects. 3.1 and 3.2) and $\mathrm{MDM}_{N}$ is the mean deviation from the median. We note that in principle $m_{\mathrm{BS}}-m_{\mathrm{tip}}$ cannot be negative, so the error bar may be asymmetric. However, the assumption of a symmetric error, although it implies a small overestimation of the left side of the uncertainty, has a negligible impact on the final results. For practical purposes, good approximations of median, standard deviation, and mean deviation from the median can be obtained by means of the following equations (valid for $50 \leq N \leq 300$ ):

$$
\begin{aligned}
& \langle\delta\rangle_{N}=0.03824 \times x^{3}-0.1627 \times x^{2}+0.09774 \times x+0.179 \\
& \operatorname{STD}_{N}=0.07502 \times x^{3}+0.57441 \times x^{2}-1.51546 \times x+1.390 \\
& \operatorname{MDM}_{N}=-0.07664 \times x^{3}+0.56869 \times x^{2}-1.43798 \times x+1.24946,
\end{aligned}
$$

$8 \mathrm{MDM}_{N}=\sum_{i} \frac{\left|\delta_{i}-\langle\delta\rangle_{N}\right|}{n}$, where $\delta_{i}$ is the brightest star-to-tip difference for the $i \mathrm{SCMD}, n$ is the total number of SCMDs, and $N$ is the number of stars in the brightest 2.5 mag of the RGB. 
where $x=\log (N)$. We note that the photometric samples of all 22 of the GGCs we analyzed contain between 50 and 200 stars in the brightest $2.5 \mathrm{mag}$ of the RGB. Clusters with $N<50$ have been excluded.

\subsection{Cluster parameters: Distance, age, and chemical composition}

Comparisons of models with observations require the knowledge of stellar parameters, such as the mass and the initial chemical composition. As is well known, globular cluster stars show substantial deviations from a scaled-solar composition. In particular, $\alpha$ elements, such as $\mathrm{O}, \mathrm{Mg}, \mathrm{Si}$, and $\mathrm{Ca}$, are enhanced $([\alpha / \mathrm{Fe}]>$ $0)$. According to Salaris et al. (1993), reliable predictions of the observed stellar properties of $\alpha$-enhanced globular cluster stars, such as the RGB tip luminosity, can be obtained with models computed assuming a scaled-solar composition with global metallicity ${ }^{9}$ :

$\left[\frac{M}{H}\right]=\left[\frac{\mathrm{Fe}}{\mathrm{H}}\right]+\log \left(a \times f_{\alpha}+b\right)$,

where $f_{\alpha}=10^{[\alpha / \mathrm{Fe}]}$ is the $\alpha$-enhancement factor, while $a$ and $b$ are numerical coefficients that depend on the adopted solar composition. According to the extant spectroscopic analysis of globular cluster stars, the average $\alpha$-enhancement is $[\alpha / \mathrm{Fe}] \sim 0.3$, with the most metal-rich clusters showing lower values, and the most metal-poor ones presenting larger enhancements (Carney 1996; Pritzl et al. 2005). In the present work, we adopted the $[\mathrm{Fe} / \mathrm{H}]$ from Carretta et al. (2009) and assumed $[\alpha / \mathrm{Fe}]=0.4$, for clusters with $[\mathrm{Fe} / \mathrm{H}]<-2,[\alpha / \mathrm{Fe}]=0.3$, for those with $-2<[\mathrm{Fe} / \mathrm{H}]<-0.8$, and $[\alpha / \mathrm{Fe}]=-0.375[\mathrm{Fe} / \mathrm{H}]$, for the more metal-rich ones. Since we adopted the Lodders et al. (2009) solar composition, the numerical coefficients should be: $a=0.6695$ and $b=0.3305$ (see Piersanti et al. 2007). Owing to the large uncertainty of the metallicity estimation, we assumed a conservative $[M / H]$ error bar of \pm 0.2 . As reported in Table 5, the propagation of this error into the tip bolometric magnitude produces an uncertainty of about \pm 0.04 mag. The estimated $[M / H]$ value of the 22 clusters in our sample are listed in Col. 2 of Table 6.

Concerning the cluster age, we assumed $13 \pm 2$ Gyr. Then, for each metallicity, we selected the initial stellar mass corresponding to the assumed age. We note that this uncertainty of the age/mass implies a marginal variation of the estimated tip bolometric magnitude (see Table 5).

For the bulk of the halo stellar population, we assumed a He abundance $Y=0.25 \pm 0.01$ (Izotov et al. 2014; Aver et al. 2015). On the other hand, the possible presence of He-enhanced stars in the cluster samples should be also considered. According to Milone et al. (2018), the He enhancement of second generation stars in the clusters of our sample is always smaller than $\delta Y_{2 G-1 G}<0.03$, while the maximum $Y$ variation is generally smaller than $\delta Y_{\max }<0.05^{10}$. We note that the RGB tip of Heenhanced stars is fainter than that of stars with normal initial $\mathrm{He}$ abundance. In addition, if the age difference between the first and the second generation of stars is negligible, the second generation RGB stars, due to the higher initial He content, should have smaller masses. This occurrence partially counterbalances the decrease of the tip luminosity caused by the He enhancement

\footnotetext{
9 As usual, the global metallicity $([M / H])$ and the total mass fraction of the elements with atomic number $\geq 6(Z)$ are related by $[M / H]=$ $\log (Z / X)-\log (Z / X)_{\odot}$.

${ }^{10}$ A larger maximum variation (0.081) is quoted for NGC 6461, but it is affected by a large uncertainty $( \pm 0.22)$.
}

Table 5. Uncertainties of cluster parameters and their effects on the tip bolometric magnitude.

\begin{tabular}{lccc}
\hline \hline Parameter & Reference value & Uncertainty & $\delta M_{\text {bol }}{ }^{(a)}$ \\
\hline$Y$ & 0.25 & $-0.01 ;+0.05$ & 0.045 \\
{$[M / H]$} & $\mathrm{C} 2009^{(b)}$ & \pm 0.2 & 0.080 \\
Age & $13 \mathrm{Gyr}$ & $\pm 10 \%$ & 0.006 \\
\hline
\end{tabular}

Notes. ${ }^{(a)}$ Full width variation of the tip bolometric magnitude. ${ }^{(b)}$ Carretta et al. (2009). See text for more details.

in second generation stars. To quantify this effect, we calculated a few He-enhanced models by reducing the initial mass in order to keep their age equal to that of normal He stars ( $13 \mathrm{Gyr})$. In practice, assuming a maximum He variation among the stars of the same cluster of $\delta Y=0.05$, the result is that the RGB tip of the most He-enhanced stars would be 0.037 mag fainter than that with normal He. Therefore, if the stars of the first generation are not a minority, the brightest RGB stars are those with normal $\mathrm{He}$. However, in the case of an overwhelming majority of Heenhanced stars, higher statistical fluctuations of the observed tip luminosity are possible. In order to take into account such a possibility, in the analysis discussed in the next section we include an additional error affecting the tip magnitude due to the $Y$ variations illustrated here. As reported in Table 5, our conservative estimation of this error is $\sigma_{Y}=0.045$, which corresponds to the systematic shift of the tip luminosity when the He content changes from $Y=0.24$ to $Y=0.30$ (or $\delta Y=0.06$ ).

Comparisons of theoretical and observed luminosities also require the knowledge of the cluster distances. Among the classical methods used to derive the distance to clusters, those based on the location in the color-magnitude diagram of the horizontal branch are widely exploited (Harris 1996, 2010; Ferraro et al. 1999). In particular, the mean luminosity of the horizontal branch or that of the zero-age horizontal branch (ZAHB) are often adopted as calibrated candles. Since the horizontal branch luminosity depends on the chemical composition, either empirical or theoretical $M_{V}(\mathrm{ZAHB})$ - metallicity relations are used. Several alternative methods have also been investigated, such as those based on main- or white-dwarf-sequence fitting, pulsation properties of RR Lyrae stars, or orbital parameters of eclipsing binaries, even if their application is often limited to smaller subsamples of the GGCs (for a complete review of these methods, see Harris 2010). Recently, the possibility of obtaining very precise parallax measurements with the Gaia astrometric satellite has been widely anticipated (Pancino et al. 2017). After the second data release (Gaia DR2), however, it has been realized that for faint sources ( $G>14 \mathrm{mag}$ ), the Gaia parallaxes are affected by significant systematic errors. In particular, these systematics substantially affect the derivation of distance to GGCs. Nevertheless, Chen et al. (2018, CH2018) were able to obtain quite accurate distances of two GGCs, NGC 362 and 47 Tuc, taking advantage of the background stars in the Small Magellanic Cloud and quasars to account for parallax systematics. Moreover, an indirect distance estimation of a large sample of GGCs was recently obtained by Baumgardt et al. (2019, B2019). Combining proper motion and radial velocity measurements from the Gaia DR2 with ground-based measurements of line-of-sight velocities, they obtained distances by fitting $N$-body GGC models to the observed kinematic properties.

In our analysis, we make use of the distance moduli we obtained following the method described in Ferraro et al. (1999) 
Table 6. RGB tip bolometric magnitudes as obtained under different assumtion for the distance scale.

\begin{tabular}{|c|c|c|c|c|c|c|c|}
\hline & {$[M / H]$} & $m-M(\mathrm{ZAHB})$ & $m-M(\mathrm{CH} 2018)$ & $m-M(\mathrm{~B} 2019)$ & $M_{\mathrm{bol}}(\mathrm{ZAHB})$ & $M_{\mathrm{bol}}(\mathrm{CH} 2018)$ & $M_{\mathrm{bol}}(\mathrm{B} 2019)$ \\
\hline \multicolumn{8}{|c|}{ Near-IR photometry } \\
\hline M92 & $-2.05 \pm 0.20$ & $14.76 \pm 0.20$ & & $14.64 \pm 0.08$ & $-3.63 \pm 0.25$ & & $-3.51 \pm 0.16$ \\
\hline M15 & $-2.03 \pm 0.20$ & $15.14 \pm 0.20$ & & $15.05 \pm 0.03$ & $-3.53 \pm 0.24$ & & $-3.44 \pm 0.13$ \\
\hline M68 & $-1.97 \pm 0.20$ & $15.14 \pm 0.20$ & & & $-3.34 \pm 0.32$ & & \\
\hline M30 & $-2.03 \pm 0.20$ & $14.73 \pm 0.20$ & & $14.48 \pm 0.24$ & $-3.65 \pm 0.28$ & & $-3.41 \pm 0.31$ \\
\hline M55 & $-1.71 \pm 0.20$ & $13.84 \pm 0.20$ & & $13.63 \pm 0.09$ & $-3.66 \pm 0.25$ & & $-3.44 \pm 0.18$ \\
\hline$\omega$ Cen & $-1.42 \pm 0.20$ & $13.67 \pm 0.20$ & & $13.60 \pm 0.02$ & $-3.59 \pm 0.23$ & & $-3.52 \pm 0.12$ \\
\hline NGC 6752 & $-1.33 \pm 0.20$ & $13.17 \pm 0.20$ & & $13.15 \pm 0.05$ & $-3.63 \pm 0.25$ & & $-3.61 \pm 0.16$ \\
\hline M13 & $-1.36 \pm 0.20$ & $14.43 \pm 0.20$ & & $14.15 \pm 0.10$ & $-3.56 \pm 0.27$ & & $-3.28 \pm 0.20$ \\
\hline M3 & $-1.28 \pm 0.20$ & $15.02 \pm 0.20$ & & $14.88 \pm 0.10$ & $-3.59 \pm 0.24$ & & $-3.45 \pm 0.17$ \\
\hline NGC 362 & $-1.08 \pm 0.20$ & $14.64 \pm 0.20$ & $14.66 \pm 0.12$ & $14.82 \pm 0.07$ & $-3.48 \pm 0.24$ & $-3.50 \pm 0.17$ & $-3.66 \pm 0.14$ \\
\hline M5 & $-1.11 \pm 0.20$ & $14.38 \pm 0.20$ & & $14.40 \pm 0.04$ & $-3.60 \pm 0.25$ & & $-3.61 \pm 0.16$ \\
\hline NGC 288 & $-1.10 \pm 0.20$ & $14.73 \pm 0.20$ & & $15.03 \pm 0.07$ & $-3.77 \pm 0.24$ & & $-4.07 \pm 0.16$ \\
\hline M107 & $-0.81 \pm 0.20$ & $13.94 \pm 0.20$ & & $13.86 \pm 0.14$ & $-3.55 \pm 0.32$ & & $-3.47 \pm 0.28$ \\
\hline NGC 6380 & $-0.29 \pm 0.20$ & $14.65 \pm 0.20$ & & & $-3.97 \pm 0.24$ & & \\
\hline NGC 6342 & $-0.36 \pm 0.20$ & $14.54 \pm 0.20$ & & & $-3.76 \pm 0.27$ & & \\
\hline 47 Tuc & $-0.55 \pm 0.20$ & $13.25 \pm 0.20$ & $13.24 \pm 0.06$ & $13.24 \pm 0.02$ & $-3.78 \pm 0.23$ & $-3.77 \pm 0.13$ & $-3.77 \pm 0.12$ \\
\hline M69 & $-0.43 \pm 0.20$ & $14.59 \pm 0.20$ & & & $-3.53 \pm 0.24$ & & \\
\hline NGC 6441 & $-0.32 \pm 0.20$ & $15.56 \pm 0.20$ & & $15.36 \pm 0.03$ & $-3.96 \pm 0.24$ & & $-3.77 \pm 0.13$ \\
\hline NGC 6624 & $-0.31 \pm 0.20$ & $14.55 \pm 0.20$ & & $14.21 \pm 0.13$ & $-3.90 \pm 0.26$ & & $-3.56 \pm 0.21$ \\
\hline NGC 6440 & $-0.15 \pm 0.20$ & $14.37 \pm 0.20$ & & & $-4.00 \pm 0.24$ & & \\
\hline NGC 6553 & $-0.12 \pm 0.20$ & $13.36 \pm 0.20$ & & $14.19 \pm 0.08$ & $-3.93 \pm 0.25$ & & $-4.76 \pm 0.17$ \\
\hline NGC 6528 & $0.07 \pm 0.20$ & $14.24 \pm 0.20$ & & & $-4.07 \pm 0.24$ & & \\
\hline \multicolumn{8}{|c|}{ VI photometry } \\
\hline NGC 362 & $-1.08 \pm 0.20$ & $14.64 \pm 0.20$ & $14.66 \pm 0.12$ & $14.82 \pm 0.07$ & $-3.51 \pm 0.26$ & $-3.53 \pm 0.21$ & $-3.69 \pm 0.17$ \\
\hline M5 & $-1.11 \pm 0.20$ & $14.38 \pm 0.20$ & & $14.40 \pm 0.04$ & $-3.63 \pm 0.26$ & & $-3.64 \pm 0.18$ \\
\hline 47 Tuc & $-0.55 \pm 0.20$ & $13.25 \pm 0.20$ & $13.24 \pm 0.06$ & $13.24 \pm 0.02$ & $-3.80 \pm 0.26$ & $-3.79 \pm 0.17$ & $-3.79 \pm 0.16$ \\
\hline
\end{tabular}

to determine the observed ZAHB luminosity. However, at variance with Ferraro et al. (1999), we used a semi-empirical $M_{V}(\mathrm{ZAHB})$ - metallicity relation. In practice, we adopted the relation reported in Eq. (4) of Ferraro et al. (1999) to describe the quadratic dependence of $M_{V}$ (ZAHB) from the metallicity, but the zero point was re-calibrated in such a way so as to reproduce the absolute $M_{V}$ (ZAHB) of NGC 362 and 47 Tuc, for which precise distances from Gaia parallaxes are available. A weighted average of the two zero points was adopted: namely, $1.03 \pm 0.07$ mag.

The distance moduli of the 22 clusters in our sample are reported in Table 6. In particular, Column 3 features the distance moduli based on the observed ZAHB luminosity, while in Cols. 4 and 5, one can see those based on Gaia parallax and kinematic measurements, as obtained by Chen et al. (2018) and Baumgardt et al. (2019), respectively. We note that for the ZAHB distances, we assumed a conservative common error of $\pm 0.2 \mathrm{mag}$, even if it may be smaller for some clusters. It includes the uncertainties in the determination of the ZAHB V magnitudes and the one due to the adopted zero point of the $M_{V}(\mathrm{ZAHB})$ - metallicity relation.

\section{Results}

The RGB tip bolometric magnitude of 22 GGCs, as estimated under different assumptions for the distances and the photometric samples, are listed in Cols. 6-8 of Table 6. Here, the $1 \sigma$ error includes the uncertainty due to; (i) the observed apparent magnitude (Sects. 3.1 and 3.2), (ii) the statistical fluctuations of the magnitudes of the brightest RGB stars (Sect. 3.4), and (iii) the cluster distance (Sect. 3.5). Figure 9 compares the tip bolometric magnitudes obtained by assuming the distance moduli based on the observed ZAHB with those derived by means of the kinematic properties. With a few exceptions, the differences are generally smaller than the quoted errors. For 47 Tuc, which is one of the two clusters for which we have the most complete sample of bright stars, that is, VIJHK data of about 200 stars in the brightest 2.5 magnitude of the RGB, the kinematic distance modulus practically coincides with that obtained from the Gaia parallax (and from the ZAHB luminosity), while for NGC 362 it is 0.16 mag larger. For the third best studied cluster, M5, we do not have a reliable estimation of the parallax, but the distance estimated from the ZAHB is in excellent agreement with that obtained by fitting the kinematic properties. Observed and predicted tip bolometric magnitudes are compared in Fig. 10. The black solid line represents our theoretical prediction for $g_{13}=0$ (no axions), while the dotted line is the linear regression of the 22 data points (squares). The latter is about 0.04 mag brighter than the theoretical expectation (at $[M / H]=-1$ ). This difference could be interpreted as a hint in favor of a small extra cooling, even if it is of the same order of magnitude of the estimated theoretical error. In any case, the axion-electron coupling should be very weak. The theoretical expectation for $g_{13}=4$ is also shown (black dashed line). We note that this value of the axion-electron coupling is indicative of the previous upper bound obtained by Viaux et al. (2013a). The observed tip bolometric magnitudes clearly exclude such a large coupling.

In order to estimate the most probable value of $g_{13}$ and its upper bound, we have calculated, for each cluster in our sample, 


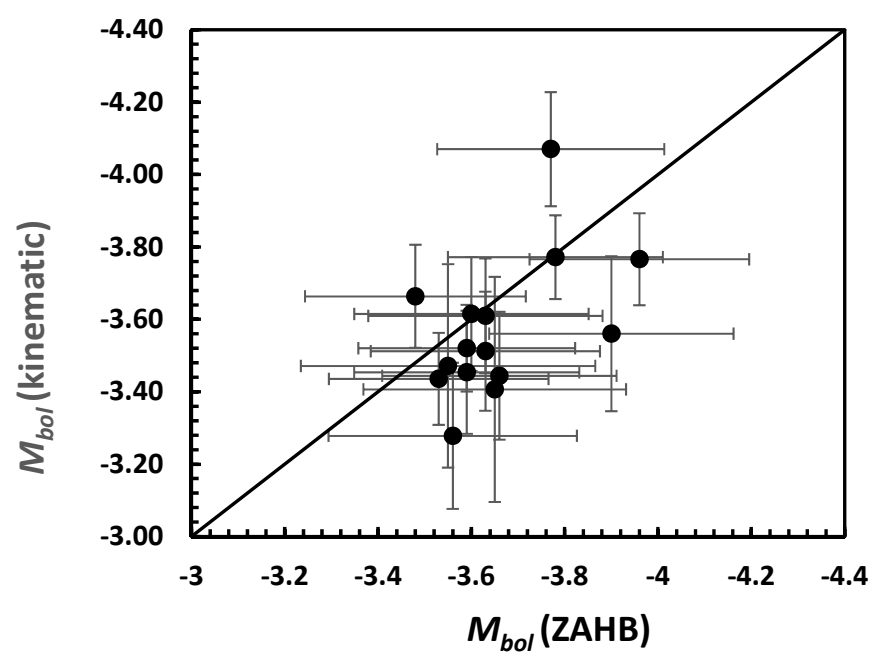

Fig. 9. Tip bolometric magnitudes of 16 GGCs obtained by assuming the ZAHB distance scale (horizontal axis) are compared to those obtained assuming the kinematic distances (verical axis), as derived by Baumgardt et al. (2019).

the following probability functions:

$l_{j}=\frac{1}{A_{j}} f_{j}$,

where

$f_{j}=\exp \left[-\frac{\left(M_{\mathrm{obs}}-M_{\text {theo }}\left(g_{13}\right)\right)^{2}}{\sigma_{\mathrm{obs}}^{2}+\sigma_{\text {theo }}^{2}+\sigma_{\mathrm{age}}^{2}+\sigma_{Y}^{2}+\sigma_{[M / H]}^{2}}\right]$,

and

$A_{j}=\int_{0}^{\infty} f_{j} d g_{13}$,

is a normalization factor. Here, $\sigma_{\text {obs }}$ are the errors of the tip absolute magnitude reported in Table 6, while $\sigma_{\text {theo }}$ represents the cumulative theoretical uncertainty (see Sect. 2.1). In addition, we also considered the uncertainties on the chemical composition, that is, $Y$ and $[M / H]$, and that on the cluster age (see Sect. 3.5 and Table 5). Then, the cumulative likelihood is:

$L\left(g_{13}\right)=\frac{1}{A} \exp \left(-\Delta^{\mathbf{T}} \mathbf{C}^{-\mathbf{1}} \boldsymbol{\Delta}\right)$,

where $\Delta$ is a vector containing the difference between the observed and the predicted tip bolometric magnitude, and $C$ is the error matrix. The latter has been computed following the procedure described in D'Agostini (1994). In particular, we considered the covariance due to the correlations introduced by the calibration of the zero point of the distance scale and bolometric corrections, as well as that due to the theoretical uncertainties (see Sects. 2.3 and 3.5).

The $l_{j}$ probability functions for the three best studied clusters (M5, 47 Tuc, and NGC362) are shown in Fig. 11. We note that for these three clusters, both optical and near-IR photometric samples are available. Furthermore, the observed tip bolometric magnitude adopted here is a weighted average of those independently obtained from the $V I$ and the $J K$ samples. Similarly, the cumulative likelihood functions, as obtained by assuming the ZAHB distances (22 clusters, solid line) or the kinematic distances (16 clusters, dashed line) are shown in Fig. 12.

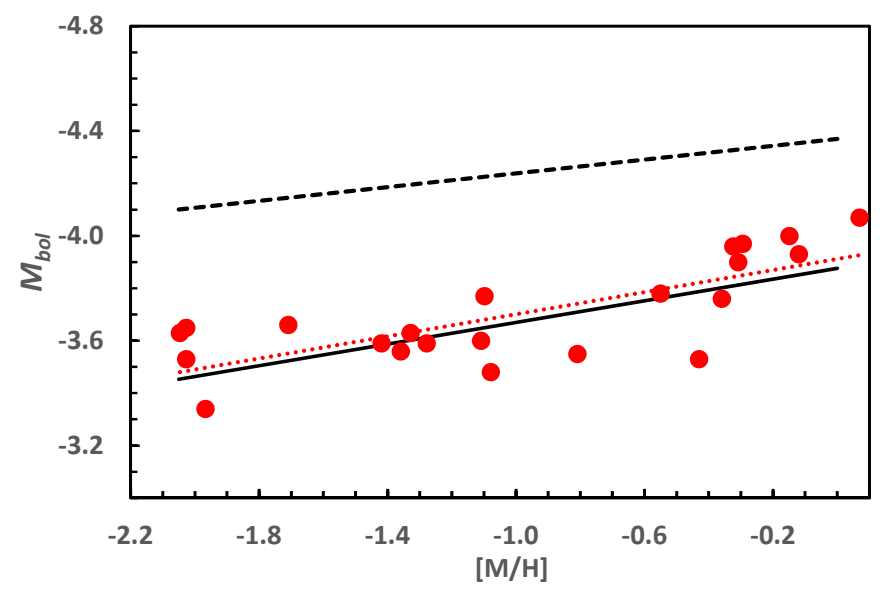

Fig. 10. Tip bolometric magnitude derived from optical and IR photometry of 22 GGCs (red circles) are compared to our theoretical predictions for $g_{13}=0$ (black solid line) and $g_{13}=4$ (black dashed line). The red dotted line represents the least-squares fit of the 22 observed $M_{\mathrm{bol}}$.

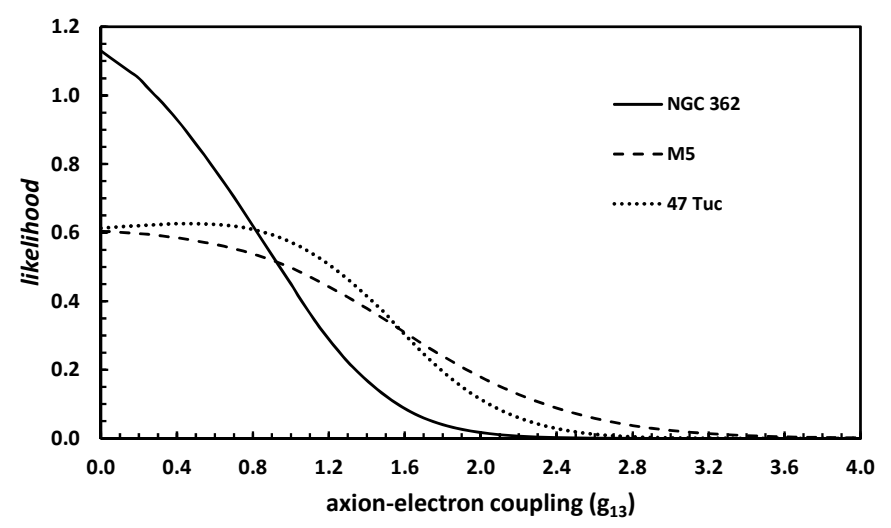

Fig. 11. Likelihood functions for M5, 47 Tuc, and NGC 362.

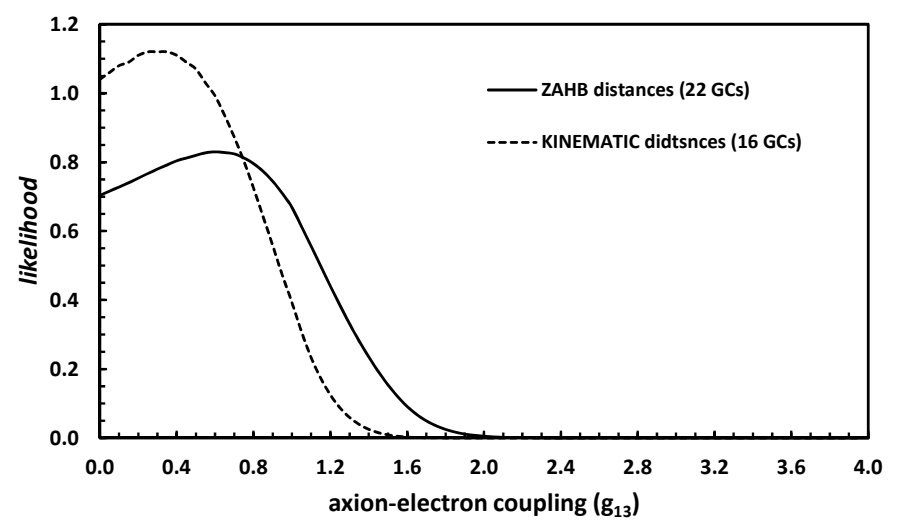

Fig. 12. Cumulative likelihood functions, as obtained under different assumptions for the distance scale.

The best values and the upper bounds for the axion-electron coupling $\left(g_{13}\right)$ are reported in Cols. 4 and 5 of Table 7. The upper bounds were obtained by imposing the conditions:

$\int_{u . b .}^{\infty} l_{j} d g_{13}=0.05$ or $\int_{u . b .}^{\infty} L d g_{13}=0.05$

where $u . b$. is the $g_{13}$ upper bound. In practice, values of $g_{13}$ greater than u.b. are excluded with $95 \%$ confidence. 
Table 7. Results of likelihood analysis.

\begin{tabular}{lcccc}
\hline \hline & $\begin{array}{c}\text { Photometric } \\
\text { sample }\end{array}$ & $\begin{array}{c}\text { Distance } \\
\text { scale }\end{array}$ & $\begin{array}{c}g_{13} \\
\text { best value }\end{array}$ & $\begin{array}{c}g_{13} \\
\text { bound }\end{array}$ \\
\hline M5 & $V I+J K$ & ZAHB & 0 & 2.30 \\
47 Tuc & $V I+J K$ & Parallax & 0.45 & 1.87 \\
NGC 362 & $V I+J K$ & Parallax & 0 & 1.37 \\
22 GGCs & $J K$ & ZAHB & 0.60 & 1.48 \\
16 GGCs & $J K$ & KINEMATIC & 0.35 & 1.15 \\
\hline
\end{tabular}

Notes. The most probable values of the $g_{13}$ parameter are listed in Col. 4 , while the corresponding upper bounds ( $95 \%$ confidence) are in Col. 5. The first three rows report the results obtained for the three best studied clusters, those for which we have used both optical and nearIR catalogs, while the last two rows list the results of the cumulative analysis. Different distance scales have been used. In particular: ZAHB stays for distances based on the observed ZAHB luminosity, (2) parallax indicates distances based on Gaia DR2 parallaxes (Chen et al. 2018) and (3) kinematic indicates distances based on the best-fit of the Gaia DR2 kinematic properties (Baumgardt et al. 2019).

The two distance scales lead to a similar conclusion: namely, there is a hint of a weak axion-electron coupling, $g_{13} \sim 0.05$, with a stringent upper bound of $g_{13}<1.5$ (95\% confidence).

\section{Conclusions}

Based on VIJK photometries of bright RGB stars and state-ofthe-art distance and metallicity scales, we determined the absolute magnitude of the RGB tip for a sample of 22 GGCs. An accurate evaluation of all the uncertainties was performed. We also revised the corresponding model predictions by analyzing the present theoretical and experimental knowledge of the relevant input physics, such as nuclear reactions, neutrino emission rates, thermodynamic properties of stellar plasma and the like. These theoretical predictions are, in general, in good agreement with the observed tip bolometric magnitudes, even if the latter are $\sim 0.04$ mag brighter, on average. This small shift between theoretical and observed tip bolometric magnitude could be the consequence of a weak axion-electron coupling. Following this hypothesis, we computed additional stellar models by including the production of thermal axions. We note that due to the high electron degeneracy, Bremsstrahlung is the most relevant thermal process capable of producing a sizeable flux of axions from the core of RGB stars. Therefore, by means of a cumulative likelihood analysis, as obtained considering all 22 clusters of our sample, we estimated the coupling parameter that determines the strength of the energy sink induced by the production of Bremsstrahlung axion. We find that the likelihood probability is maximized for $g_{a e} \sim 0.60_{-0.58}^{+0.32} \times 10^{-13}$. Moreover, we find an upper bound for this parameter of $g_{a e}=1.48 \times 10^{-13}$, with $95 \%$ confidence.

This new bound represents the more stringent constraint for the axion-electron coupling available so far. Indeed, in addition to the work of Viaux et al. (2013a), who reported $g_{a e}<$ $4.3 \times 10^{-13}$ from the luminosity of the RGB tip of the globular cluster M5, other astrophysical constraints we find in the extant literature are those of Miller Bertolami et al. (2014, see also Isern 2018), reporting $g_{a e}<2.8 \times 10^{-13}$, as obtained from an analysis of the white dwarf (WD) luminosity functions, and Córsico et al. (2016), who found $g_{a e}<7 \times 10^{-13}$, from the period drift of pulsating WDs.
Concerning the direct search for axions, recent years have seen impressive experimental efforts (for a review of the axion experimental landscape, see Irastorza \& Redondo 2018; DiLuzio et al. 2020b, and Sikivie 2020). However, the axion coupling to electrons is particularly difficult to probe experimentally. The most stringent upper bounds have been obtained by the XENON100 collaboration (Aprile et al. 2014), $g_{a e}<7.7 \times 10^{-12}$ (90\% CL), LUX (Akerib 2017), $g_{a e}<3.5 \times 10^{-12}$, and PandaXII (Fu et al. 2017), $g_{a e}<4 \times 10^{-12}$. These bounds are not yet competitive with the stellar bounds on this coupling.

Before submitting the present paper, there was an announcement from the XENON1T collaboration of a possible detection of solar axions (Aprile et al. 2020). For values of the axionphoton coupling not excluded by previous experiments (e.g., Anastassopoulos et al. 2017), they find $27<g_{a e} / 10^{13}<37$ (90\% confidence), a value at least 20 times higher than the upper bound we obtained in this work (see also Di Luzio et al. 2020a). Alternative interpretations of the signal detected by XENON1T have already been proposed (see the discussion in Aprile et al. 2020), among which the possibility that this signal is due to a dark matter ALP with mass of a few $\mathrm{keV}$ and a coupling-toelectron $g_{a e} \sim 10^{-13}$ (Takahashi et al. 2020). In that case, these ALPs may constitute all or a fraction of the local dark matter. Such an explanation would remove the tension of the XENON1T detection with our work.

Finally, we remark that the present upper bound for the axion-electron coupling remains valid also in the case of additional energy sinks active during the RGB evolutionary phase, not included in the present models, such as a nonzero neutrino magnetic moment or a sizeable axion coupling with photons. On the contrary, the existence of these additional energy sinks would affect the hint on $g_{a e}$. For example, in Sect. 2.2, we recall that at the high density of the core of an RGB star, the Primakoff process is suppressed. Nevertheless, its effect on the RGB tip luminosity may be not completely negligible. In the most extreme case of an axion-photon coupling of $g_{a \gamma}=6 \times 10^{-11} \mathrm{GeV}^{-1}$, that is, the upper bound independently obtained from the solar axions by the CAST collaboration (Anastassopoulos et al. 2017) and from the horizontal branch lifetimes (Ayala et al. 2014), the resulting upward shift of the RGB tip would explain most of the small difference between the standard theoretical predictions (no axions) and the least-squares fit of the observed tip luminosity (the solid and the dotted curves in Fig. 10). In this framework, we cannot exclude a much smaller (at least 0) axion-electron coupling.

Acknowledgements. We are in debt with F. Capozzi and G. Raffelt for a careful reading of our manuscript. We acknowledge that after our submission to A\&A, they uploaded on the ArXiv an independent study where they reached conclusions similar to ours. Our work has been supported by the Agenzia Spaziale Italiana (ASI) and the Instituto Nazionale di Astrofsica (INAF) under the agreement n. 2017-14-H.0 - attività di studio per la comunità scientifica di Astrofisica delle Alte Energie e Fisica Astroparticellare. I.D. research is also supported by the MICINN-FEDER project PGC2018-095317-B-C21, while A. M. is partially supported by the Istituto Nazionale di Fisica Nucleare (INFN), through the "Theoretical Astroparticle Physics" project, and by the research grant number 2017W4HA7S: "Neutrino and Astroparticle Theory Network", under the program PRIN 2017 funded by the Italian Ministero dell'Universitá e della Ricerca (MUR).

\section{References}

Adelberger, E. G., García, A., Robertson, R. G. H., et al. 2011, Rev. Mod. Phys., 83,195

Akerib, D. S., Alsum, S., Aquino, C., et al. 2017, Phys. Rev. Lett., 118, 261301 Anastassopoulos, V., Aune, S., Barth, K., et al. 2017, Nat. Phys., 13, 584 
Angulo, C., Arnould, M., Rayet, M., et al. 1999, Nucl. Phys. A, 656, 3 Aprile, E., Agostini, F., Alfonsi, M., et al. 2014, Phys. Rev. D, 90, 062009 Aprile, E., Aalbers, J., Agostini, F., et al. 2020, Phys. Rev. D, 102, 072004 Aver, E., Olive, K. A., \& Skillman, E. D. 2015, J. Cosmol. Astropart. Phys., 2015, 011

Ayala, A., Domínguez, I., Giannotti, M., Mirizzi, A., \& Straniero, O. 2014, Phys Rev. Lett., 113, 191302

Baumgardt, H., Hilker, M., Sollima, A., \& Bellini, A. 2019, MNRAS, 482, 5138

Beaudet, G., Petrosian, V., \& Salpeter, E. E. 1967, ApJ, 150, 979

Beccari, G., Ferraro, F. R., Lanzoni, B., \& Bellazzini, M. 2006, ApJ, 652, L121

Buzzoni, A., Patelli, L., Bellazzini, M., Pecci, F. F., \& Oliva, E. 2010, MNRAS 403, 1592

Cadelano, M., Dalessandro, E., Ferraro, F. R., et al. 2017, ApJ, 836, 170

Cardelli, J. A., Clayton, G. C., \& Mathis, J. S. 1989, ApJ, 345, 245

Carney, B. W. 1996, PASP, 108, 900

Carretta, E., Bragaglia, A., Gratton, R., D’Orazi, V., \& Lucatello, S. 2009, A\&A, 508,695

Chaboyer, B., Deliyannis, C. P., Demarque, P., et al. 1992, ApJ, 388, 372

Chen, S., Richer, H., Caiazzo, I., \& Heyl, J. 2018, ApJ, 867, 132

Córsico, A. H., Romero, A. D., Althaus, L. G., et al. 2016, JCAP, 7, 036

Cox, J., \& Giuli, R. 1968, Principles of Stellar Structure: Applications to Stars, Principles of Stellar Structure (Gordon and Breach)

Cyburt, R. H., Amthor, A. M., Ferguson, R., et al. 2010, ApJS, 189, 240

D’Agostini, G. 1994, Nucl. Instrum. Methods Phys. Res. A, 346, 306

Dalessandro, E., Pallanca, C., Ferraro, F. R., et al. 2014, ApJ, 784, L29

Delahaye, F., \& Pinsonneault, M. 2005, ApJ, 625, 563

Dell'Omodarme, M., Valle, G., Degl'Innocenti, S., \& Prada Moroni, P. G. 2012, A\&A, 540, A26

Dewitt, H. E., Graboske, H. C., \& Cooper, M. S. 1973, ApJ, 181, 439

Dicus, D. A., Kolb, E. W., Schramm, D. N., \& Tubbs, D. L. 1976, ApJ, 210 481

Di Luzio, L., Fedele, M., Giannotti, M., Mescia, F., \& Nardi, E. 2020a, Phys. Rev. Lett., 125, 131804

Di Luzio, L., Giannotti, M., Nardi, E., \& Visinelli, L. 2020b, Phys. Rep., 870, 1

Ferraro, F. R., Messineo, M., Fusi Pecci, F., et al. 1999, AJ, 118, 1738

Ferraro, F. R., Montegriffo, P., Origlia, L., \& Fusi Pecci, F. 2000, AJ, 119, 1282

Formicola, A., Imbriani, G., Costantini, H., et al. 2004, Phys. Lett. B, 591, 61

Freedman, W. L., Madore, B. F., Hoyt, T., et al. 2020, ApJ, 891, 57

Fu, C., Zhou, X., Chen, X., et al. 2017, Phys. Rev. Lett., 119, 181806

Fynbo, H. O. U., Diget, C. A., Bergmann, U. C., et al. 2005, Nature, 433, 136

Goriely, S., Hilaire, S., \& Koning, A. J. 2008, A\&A, 487, 767

Graboske, H. C., Dewitt, H. E., Grossman, A. S., \& Cooper, M. S. 1973, ApJ, 181,457

Haft, M., Raffelt, G., \& Weiss, A. 1994, ApJ, 425, 222

Harris, W. E. 1996, AJ, 112, 1487

Harris, W. E. 2010, ArXiv e-prints [arXiv:1012.3224]

Iglesias, C. A., \& Rogers, F. J. 1996, ApJ, 464, 943

Imbriani, G., Costantini, H., Formicola, A., et al. 2004, A\&A, 420, 625

Irastorza, I. G., \& Redondo, J. 2018, Progress in Particle and Nuclear Physics, 102,89

Itoh, N., Totsuji, H., Ichimaru, S., \& Dewitt, H. E. 1979, ApJ, 234, 1079

Itoh, N., Hayashi, H., Nishikawa, A., \& Kohyama, Y. 1996, ApJS, 102, 411

Izotov, Y. I., Thuan, T. X., \& Guseva, N. G. 2014, MNRAS, 445, 778

Kiss, L. L., \& Bedding, T. R. 2003, MNRAS, 343, L79

Krishna Swamy, K. S. 1966, ApJ, 145, 174

Lederer, M. T., \& Aringer, B. 2009, A\&A, 494, 403
Lemut, A., Bemmerer, D., Confortola, F., et al. 2006, Phys. Lett. B, 634, 483

Lodders, K., Palme, H., \& Gail, H. P. 2009, Landolt Börnstein, 712

Miller Bertolami, M. M., Melendez, B. E., Althaus, L. G., \& Isern, J. 2014, JCAP, 10, 069

Milone, A. P., Marino, A. F., Renzini, A., et al. 2018, MNRAS, 481, 5098

O'Donnell, J. E. 1994, ApJ, 422, 158

Pallanca, C., Lanzoni, B., Dalessandro, E., et al. 2013, ApJ, 773, 127

Pallanca, C., Ransom, S. M., Ferraro, F. R., et al. 2014, ApJ, 795, 29

Pallanca, C., Ferraro, F. R., Lanzoni, B., et al. 2019, ApJ, 882, 159

Pancino, E., Bellazzini, M., Giuffrida, G., \& Marinoni, S. 2017, MNRAS, 467, 412

Peccei, R. D., \& Quinn, H. R. 1977, Phys. Rev. Lett., 38, 1440

Piersanti, L., Straniero, O., \& Cristallo, S. 2007, A\&A, 462, 1051

Piersanti, L., Cristallo, S., \& Straniero, O. 2013, ApJ, 774, 98

Pietrinferni, A., Cassisi, S., Salaris, M., \& Castelli, F. 2004, ApJ, 612, 168

Potekhin, A. Y. 1999, A\&A, 351, 787

Potekhin, A. Y., Baiko, D. A., Haensel, P., \& Yakovlev, D. G. 1999, A\&A, 346, 345

Prada Moroni, P. G., \& Straniero, O. 2002, ApJ, 581, 585

Pritzl, B. J., Venn, K. A., \& Irwin, M. 2005, AJ, 130, 2140

Proffitt, C. R., \& Vandenberg, D. A. 1991, ApJS, 77, 473

Raffelt, G. G. 1996, Stars as Laboratories for Fundamental Physics: The Astrophysics of Neutrinos, Axions, and Other Weakly Interacting Particles (University of Chicago Press) QB464.2.R34 1996

Raffelt, G., \& Weiss, A. 1995, Phys. Rev. D, 51, 1495

Rogers, F. J., Swenson, F. J., \& Iglesias, C. A. 1996, ApJ, 456, 902

Salaris, M., Chieffi, A., \& Straniero, O. 1993, ApJ, 414, 580

Sallaska, A. L., Iliadis, C., Champange, A. E., et al. 2013, ApJS, 207, 18

Salpeter, E. E. 1954, Aust. J. Phys., 7, 373

Sarajedini, A., Bedin, L. R., Chaboyer, B., et al. 2007, AJ, 133, 1658

Serenelli, A., Weiss, A., Cassisi, S., Salaris, M., \& Pietrinferni, A. 2017, A\&A, 606, A33

Sikivie, P. 2020, Rev. Mod. Phys., submitted [arXiv:2003.02206]

Sollima, A., Ferraro, F. R., Origlia, L., Pancino, E., \& Bellazzini, M. 2004, A\&A, 420,173

Stetson, P. B. 1987, PASP, 99, 191

Stetson, P. B., Pancino, E., Zocchi, A., Sanna, N., \& Monelli, M. 2019, MNRAS, 485, 3042

Straniero, O. 1988, A\&AS, 76, 157

Straniero, O., Chieffi, A., \& Limongi, M. 1997, ApJ, 490, 425

Straniero, O., Gallino, R., \& Cristallo, S. 2006, Nucl. Phys. A, 777, 311

Straniero, O., Dominguez, I., Piersanti, L., Giannotti, M., \& Mirizzi, A. 2019, ApJ, 881, 158

Takahashi, F., Yamada, M., \& Yin, W. 2020, Phys. Rev. Lett., 125, 161801

Thoul, A. A., Bahcall, J. N., \& Loeb, A. 1994, ApJ, 421, 828

Turcotte, S., Richer, J., Michaud, G., et al. 1998, ApJ, 504, 539

Valcarce, A. A. R., Catelan, M., \& Sweigart, A. V. 2012, A\&A, 547, A5

Valenti, E., Ferraro, F. R., \& Origlia, L. 2004a, MNRAS, 351, 1204

Valenti, E., Ferraro, F. R., Perina, S., \& Origlia, L. 2004b, A\&A, 419, 139

Valle, G., Dell'Omodarme, M., Prada Moroni, P. G., \& Degl'Innocenti, S. 2013, A\&A, 549, A50

Viaux, N., Catelan, M., Stetson, P. B., et al. 2013a, Phys. Rev. Lett., 111, 231301

Viaux, N., Catelan, M., Stetson, P. B., et al. 2013b, A\&A, 558, A12

Weinberg, S. 1978, Phys. Rev. Lett., 40, 223

Wilczek, F. 1978, Phys. Rev. Lett., 40, 279

Worthey, G., \& Lee, H.-C. 2011, ApJS, 193, 1

Xu, Y., Takahashi, K., Goriely, S., et al. 2013, Nucl. Phys., A, 918 